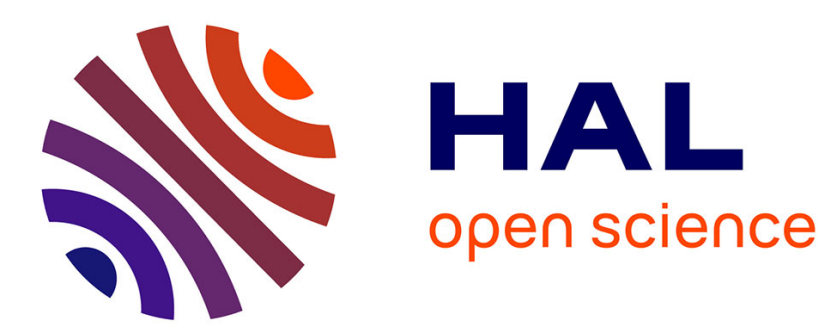

\title{
High temperature damage model for carbon-carbon composites
}

\author{
Patrick Laborde, B. Toson, Marc Odunlami
}

\section{To cite this version:}

Patrick Laborde, B. Toson, Marc Odunlami. High temperature damage model for carbon-carbon composites. European Journal of Mechanics - A/Solids, 2011, 10.1016/j.euromechsol.2010.12.014 . hal-00734540

\section{HAL Id: hal-00734540 \\ https://hal.science/hal-00734540}

Submitted on 23 Sep 2012

HAL is a multi-disciplinary open access archive for the deposit and dissemination of scientific research documents, whether they are published or not. The documents may come from teaching and research institutions in France or abroad, or from public or private research centers.
L'archive ouverte pluridisciplinaire HAL, est destinée au dépôt et à la diffusion de documents scientifiques de niveau recherche, publiés ou non, émanant des établissements d'enseignement et de recherche français ou étrangers, des laboratoires publics ou privés. 


\section{Accepted Manuscript}

Title: High temperature damage model for carbon-carbon composites

Authors: P. Laborde, B. Toson, M. Odunlami

PII: S0997-7538(10)00152-X

DOI: $\quad$ 10.1016/j.euromechsol.2010.12.014

Reference: EJMSOL 2669

To appear in: European Journal of Mechanics / A Solids

Received Date: 3 December 2009

Revised Date: 13 December 2010

Accepted Date: 22 December 2010

Please cite this article as: Laborde, P., Toson, B., Odunlami, M. High temperature damage model for carbon-carbon composites, European Journal of Mechanics / A Solids (2010), doi: 10.1016/ j.euromechsol.2010.12.014

This is a PDF file of an unedited manuscript that has been accepted for publication. As a service to our customers we are providing this early version of the manuscript. The manuscript will undergo copyediting, typesetting, and review of the resulting proof before it is published in its final form. Please note that during the production process errors may be discovered which could affect the content, and all legal disclaimers that apply to the journal pertain. 


\title{
High temperature damage model for carbon-carbon composites
}

\author{
P. Laborde ${ }^{\mathrm{a}, *}$, B. Toson ${ }^{\mathrm{b}}$, M. Odunlami ${ }^{\mathrm{c}}$ \\ ${ }^{a}$ Université de Toulouse, CNRS, Université Paul Sabatier, Institut de Mathématiques de \\ Toulouse, 118 route de Narbonne, F-31062 Toulouse Cedex 9 \\ ${ }^{b}$ CEA, DAM, CESTA, F-33114 Le Barp \\ ${ }^{c}$ Laboratoire de Mathématiques et de leurs Applications, Université de Pau et des Pays \\ de l'Adour, BP 1155, F-64013 Pau Cedex
}

\begin{abstract}
A damage model for carbon-carbon orthotropic composite materials is introduced with a special attention to the thermo-mechanical effects. The internal variables of damage are identified from tension-compression tests according to each fiber direction and from shear tests in each orthotropy plane. The influence of the temperature is taken into account from typical experimental stress-strain curves given for different values of the temperature. The finite element model was implemented in $A B A Q U S^{\circledR}$ using an implicit time incremental scheme. Finally, a significant numerical simulation of a thermomechanical loading with damage is presented.
\end{abstract}

Keywords:

carbon-carbon composites, orthotropic damage, thermo-mechanics effects

${ }^{*}$ Corresponding author. Tel.: +33 5 61556377; fax: +33561558385

Email address: patrick.laborde@math.ups-tlse.fr (P. Laborde) 


\section{Introduction}

During the last forty years, there has been a great expansion of $3 D$ carboncarbon $(C / C)$ composite materials such as AEROLOR ${ }^{\circledR}$. Industry takes advantage of their high performance thermal and mechanical properties. The $C / C$ composites are an attractive choice for aeronautics (structural components, aircraft brakes), in spatial applications (rocket thrust nozzles, hypersonic vehicles), in the domain of nuclear fusion (Tore Supra tokamak), etc. Tests in a real-life situation are costly, limited and difficult to analyze, so the prediction of the thermo-mechanical behavior of $C / C$ materials by means of numerical simulations is essential. On account of the strongly nonlinear rheology of these materials, the difficulty is to obtain reliable models associated to robust numerical schemes.

A considerable amount of works has been carried out to model damage processes for composite materials. The framework generally adopted is provided by the theory of Thermodynamics of Irreversible Processes with internal variables. Among these works, we find those from the ENS Cachan, France, with a first model proposed in the early 1980s by Poss (1981). In the 1990s, intensive developments have been achieved by numerous researchers such as Allix et al. (1993), Ladevèze et al. (1994), Gasser (1994), or Maire and Chaboche (1997), Maire and Lesne (1997), Maire et al. (1996) at ONERA (France). A hierarchical method of modelling was used for various composites such as $\mathrm{SiC} / \mathrm{SiC}, \mathrm{C} / \mathrm{SiC}$ and $\mathrm{C} / \mathrm{C}$ (Chaboche, 1997). More recently, in order to study specific models for $C / C$ composites, the concepts developed in the previous works were considered by Pailhes et al. (2002), Siron (1996), Moncel (1999) (LCTS, France). Let us also quote works on anisotropic dam- 
age models for concrete within a non standard thermodynamic framework : Ragueneau et al. (2008), Desmorat et al. (2010).

Nevertheless, most of the studies on $3 D C / C$ materials are made at the ambient temperature. In some applications, the composite material is subjected to extreme thermal variations and high temperatures significantly affect its mechanical properties. Experimental loading-unloading curves show that the dependence with respect to the temperature of the elastic stiffness and of the stress threshold may be important and strongly nonlinear. So it is necessary to take into account the coupling of damage and temperature to write the constitutive equations ; for example, the elastic stiffness will have to depend not only on the damage history but also on the current temperature. Such a behavior with damage for a varying temperature seems difficult to predict from existing works.

Our goal is to take into account the effect of the temperature on the inelastic behavior of 3D C/C by using an alternative approach. A mathematical framework of modelling is introduced having the following requirements in view. (i) The identification of the mechanical properties needs loadingunloading tests at different values of the temperature. (ii) The general model has to supply identifiable specific constitutive equations which exactly reproduce the available experimental stress-strain curves. (iii) One must be able to associate an efficient approximation scheme compatible with the architecture of standard nonlinear computational mechanics softwares.

An application is given from generic stress-strain curves corresponding to experimental data available now from uniaxial loading-unloading tests. Here, internal variables of damage are defined from the strain path and the 
elastic stiffness is identified from the set $\alpha$ of the internal variables and the temperature $\theta$. For this simplified model, an assumption of uncoupling of the damage state according to the fiber directions is used. The irreversibility of the evolution in the model is ensured by the fact that the rate of the internal variables is proved to be nonnegative. The numerical solution was performed using an implicit incremental scheme implemented in the commercial software ABAQUS. The proposed approach of modelling is general enough so that one can possibly specify more sophisticated constitutive equations from future experimental diagrams for $\mathrm{C} / \mathrm{C}$ under multiaxial thermo-mechanical loading.

The outline of the paper is as follows. Section 2 is devoted to AEROLOR ${ }^{\circledR}$ type $C / C$ composites and their main thermo-mechanical properties. We define, in the following section, a unidimensional model at a fixed temperature from a convenient choice of a damage internal variable. In section 4, a typical stress-strain curve is considered representing loading-unloading cycles in a uniaxial tensile test on a $C / C$ material. Consistency of this model with the given diagram typical of a damage behavior is proved. Again within a unidimensional framework, the following section deals with the case where the stress-strain curves depend on the temperature. Next, in section 6 , we are in a position to introduce a three-dimensional model of damage with changes in temperature. The specific features of the $C / C$ composites behavior are taken into consideration: directional uncoupling of damage, tension-compression difference of behavior, alternating shear. The next section concerns an implicit time discretization of the model; we show that the nonlinearity at each step in the incremental scheme is of projection type. In the final section, a significant computational test is presented relative to the quasi-static evolu- 


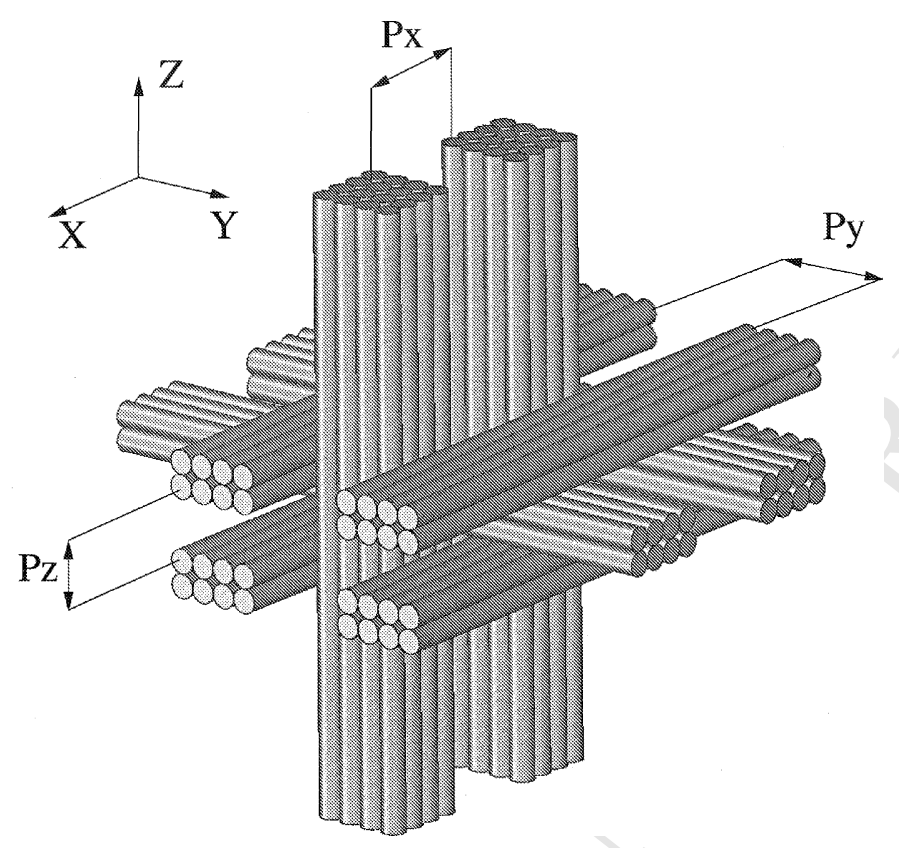

Figure 1: Architecture of the AEROLOR ${ }^{\circledR}$ material.

tion damage problem.

\section{Presentation of the $C / C$ composite}

The AEROLOR ${ }^{\circledR}$ type composite materials are constituted from a tridirectional weaving (Figure 1 ). In the $z$ direction, the strands are spaced by a pitch $P_{z}$ of $8 \times 10^{-4} \mathrm{~m}$. The other two directions are equivalent with $P_{x}=P_{y}=16 \times 10^{-4} \mathrm{~m}$. The strands oriented along $z$ are made by assembling $4 \times 4$ threads of 3000 carbon fibers. The strands oriented along $x$ and $y$ consist of $2 \times 4$ threads. The mean diameter of a fiber is $6.5 \times 10^{-6} \mathrm{~m}$ and the fiber volume ratio is identical in all directions, in the order of 15 . The woven block is densified in liquid phase. Densification includes two low pressure cycles and five high pressure cycles. Each densification cycle, except the last 
one, is followed by a graphitization procedure. In the last high pressure cycle, the material is heat treated at $1200^{\circ} \mathrm{C}$. The graphitization of the preceding five cycles is performed at $2600^{\circ} \mathrm{C}$. At the end of the elaboration process, the virgin material is free of residual stresses. This point was experimentally verified.

The main advantages of $C / C$ composite materials are the following:

- a good resistance to thermal shocks, enabling their use up to temperatures at almost $3500^{\circ} \mathrm{C}$,

- an extremely low density, between 1.5 and 2 depending on the quality of the material, enabling substantial weight-saving compared to tungsten.

- a high stiffness even at elevated temperatures,

- a good ablation resistance: the refractory properties and the high sublimation latent heat of the carbon make it the best ablation material known.

\section{Unidimensional modelling at a fixed temperature}

The study of a uniaxial tensile test on a $C / C$ composite material with loading-unloading cycles will allow us to bring out important features of the concerned behavior with damage. In the present section, the temperature is fixed; modelling tools are introduced in order to include large heat variations further down. Stress $\sigma$ and mechanical strain $\varepsilon$ are scalar quantities in this unidimensional analysis. 


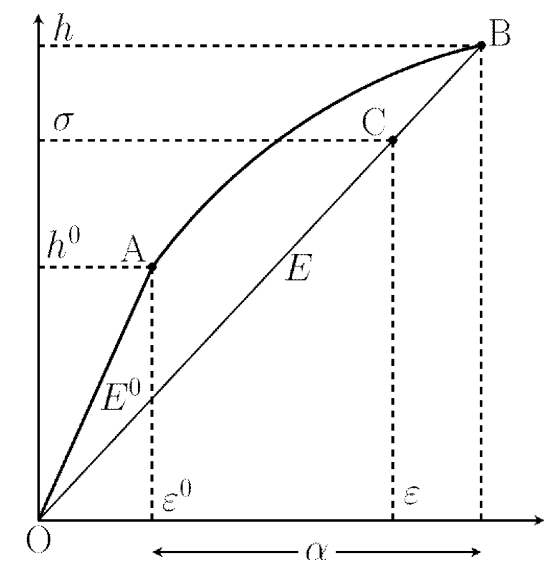

Figure 2: Uniaxial tensile test.

In the following, the quantities under study will be assumed to be smooth enough so that computations make sense. For a mathematical study of the damage problem, we refer to a forthcoming paper (Laborde, to appear).

\subsection{Typical behavior of damage}

Let us consider a stress-strain curve characteristic of the behavior of $C / C$ composite materials at a fixed temperature. In Figure 2, we present in diagram form a loading-unloading cycle for a uniaxial tensile test into the longitudinal orthotropic direction $z$. Residual strains are assumed to be negligible in this first approach. Here $\varepsilon$ denotes the longitudinal strain and $\sigma$ stands for the uniaxial stress. In order to identify the main features of the considered nonlinear behavior, we will study the case of a tensile test $(\sigma>0)$ in the following unidimensional analysis.

The line segment $O A$ of the stress-strain curve corresponds to a linear reversible behavior as long as $\varepsilon$ remains below some initial limit $\varepsilon^{0}$ (possibly equal to zero). The associated elastic stiffness is denoted $E^{0}$ and $h^{0}$ is the 
initial stress threshold of damage. The arc of a curve $A B$ points to the nonlinear damage mechanism into play at this stage of loading. The stress level $h^{0}$ at point $A$ is brought to a new value $h$ at point $B$. At the end of the elastic unloading path $B O$, let us observe that no residual strain appears in Figure 2. The current Young's modulus $E$ is lesser than the intial one $E^{0}$ and a supplementary decreasing of the Young's modulus will be possible only when the stress will pass beyond the previous threshold $h$. The weakening of the elastic stiffness is significant of damage phenomena. Figure 2 is a simplified representation of an irreversible process.

During the history of deformations at the fixed temperature, the current Young's modulus $E$ and stress threshold $h$ are measurable quantities. We put

$$
\alpha=\frac{h}{E}-\varepsilon^{0}
$$

where $\varepsilon^{0}$, possibly equal to zero, is the intial limit of reversibility for strains. Here, the terminology initial refers to a characteristic of the virgin (non damaged) material :

$$
\varepsilon^{0}=\frac{h^{0}}{F^{0}}
$$

The definition of $\alpha$ is illustrated in Figure 2.

The quantities $E$ and $h$ can be identified as a function of the variable $\alpha$

$$
E=E(\alpha), \quad h=h(\alpha)
$$

from the given experimental stress-strain curve in Figure 2. Functions $E(\alpha)$ and $h(\alpha)$ will be considered as data in the following modelling procedure, so as the value $\varepsilon^{0}$. 
The Young's modulus is assumed to be a differentiable, nonnegative and decreasing function of the variable $\alpha$ :

$$
E_{\alpha}^{\prime}<0
$$

where the prime denotes a (partial) derivative with respect to the indexed variable. We also suppose that the stress threshold is a differentiable, nonnegative and increasing function with respect to $\alpha$ as in Figure 2 :

$$
h_{\alpha}^{\prime}>0
$$

A last hypothesis is the concavity of the function $h$. From the definition (1), we deduce

$$
\frac{d}{d \alpha}\left(\frac{h}{E}-\varepsilon^{0}\right)=1
$$

Such a condition will be discussed in the general case in section 5.1.

The quantity $\alpha$ depends on the the history of deformations and defines the state of irreversibility of the system. The parameter $\alpha$ will play the role of an internal variable in the constitutive equations below. The value of $\alpha$ determines the degradation of the elastic stiffness property of the material during the loading process and $\alpha$ will be called the variable of damage.

\subsection{Constitutive equations}

From the definition of the internal variable $\alpha$ above, we are now in a position to express the irreversible process considered in Figure 2. In the previous subsection, the stress threshold $h$ was introduced so that the stress satisfies the admissibility criterion:

$$
\varphi(\sigma, \alpha) \leq 0
$$


denoting $\varphi(\sigma, \alpha)=\sigma-h(\alpha)$ in tension $(\sigma \geq 0)$. Let us note that $\varphi$ is a convex function from the concavity property of the yield function $h$. By taking into account the behavior of the internal variable illustrated in Figure 2 , we can write

$$
\dot{\alpha} \geq 0 \quad \text { and } \quad \dot{\alpha}=0 \text { if } \varphi<0
$$

The dot stands for a derivative with respect to the time $t$ (the loading parameter).

The elastic behavior is governed by the uniaxial Hooke's law

$$
\sigma=E(\alpha) \varepsilon
$$

in the framework of infinitesimal strains. By inverting the previous equality, one obtains

$$
\varepsilon=C(\alpha) \sigma
$$

were $C(\alpha)$ is the elastic compliance

$$
C(\alpha)=\frac{1}{E(\alpha)}
$$

The rate of strain is obtained by differentiating equality (10) :

$$
\dot{\varepsilon}=C \dot{\sigma}+\dot{\varepsilon}^{D},
$$

where the contribution of the damage is written

$$
\dot{\varepsilon}^{D}=C_{\alpha}^{\prime} \sigma \dot{\alpha}
$$

By putting

$$
\dot{\varepsilon}^{D}=\dot{\lambda},
$$


we derive from (8):

$$
\dot{\lambda} \geq 0 \quad \text { and } \quad \dot{\lambda}=0 \text { if } \varphi<0,
$$

thanks to the fact that the Young's modulus is a decrasing function from (4).

It follows that

$$
\left(\dot{\varepsilon}^{D},-A \dot{\alpha}\right)=\dot{\lambda} \nabla \varphi
$$

with the notation

$$
\nabla \varphi=\left(\frac{\partial \varphi}{\partial \sigma}, \frac{\partial \varphi}{\partial \alpha}\right)=\left(1,-h_{\alpha}^{\prime}\right)
$$

for the gradient of function $\varphi$ and

$$
A=C_{\alpha}^{\prime} h h_{\alpha}^{\prime}
$$

Let us observe that $A>0$ since the yield limit is a strictly increasing function from (5).

In short, the uniaxial constitutive equations are written :

$$
\begin{aligned}
& \dot{\varepsilon}=C \dot{\sigma}+\dot{\varepsilon}^{D}, \\
& \varphi(\sigma, \alpha) \leq 0, \\
& \left(\dot{\varepsilon}^{D},-A \dot{\alpha}\right)=\dot{\lambda} \nabla \varphi, \\
& \dot{\lambda} \geq 0, \quad \dot{\lambda}=0 \quad \text { if } \quad \varphi<0 .
\end{aligned}
$$

In the following section, we will show that this specific model reproduces exactly the given typical stress-strain diagram for a fixed temperature.

\section{Consistency of the model}

The representative experimental loading-unloading strain curve of Figure 2 illustrates different features of the behavior with damage. The aim is to 
verify that the previous unidimensional model fits to such a stress-strain diagram.

Proposition 1. Under the assumptions of section 3.1, let $\sigma, \varepsilon, \alpha$ be a given state such that one has $0<\sigma \leq h(\alpha)$. The stress rate response $\dot{\sigma}$ in terms of the strain rate $\dot{\varepsilon}$ is determined by the model (14) as follows.

(i) If $\sigma<h$ (elastic range) or if $\sigma=h$ and $\dot{\varepsilon} \leq 0$ (elastic unloading), then

$$
\dot{\sigma}=E(\alpha) \dot{\varepsilon}, \quad \dot{\alpha}=0 .
$$

(ii) If $\sigma=h$ and $\dot{\varepsilon}>0$, then (damage range)

$$
\dot{\sigma}=E_{T}(\alpha) \dot{\varepsilon}, \quad \dot{\alpha}=\dot{\varepsilon},
$$

where $E_{T}=h_{\alpha}^{\prime}$ is the tangent modulus.

This result shows that the model (14) is relevant to represent the given stress-strain diagram, as illustrated in Figure 3.

Remark 1. At this stage of the modelling procedure, the regularity of the functions involved in the calculations has been not specified. Actually, the correct notion of velocity is in fact given by the right-hand derivative in time:

$$
\frac{d^{+} \sigma}{d t}(t)=\lim _{h \rightarrow 0, h>0} \frac{1}{h}(\sigma(t+h)-\sigma(t)) .
$$

The existence of this quantity can be established in a suitable mathematical framework. The following demonstration also implicitly uses the fact that:

$$
\frac{d^{+} \sigma}{d t}(t)=\lim _{h \rightarrow 0, h>0} \frac{1}{h} \int_{t}^{t+h} \dot{\sigma}(t) d t
$$

where $\dot{\sigma}$ corresponds to a weak notion of the derivation in time. For further details, see Laborde (to appear). 


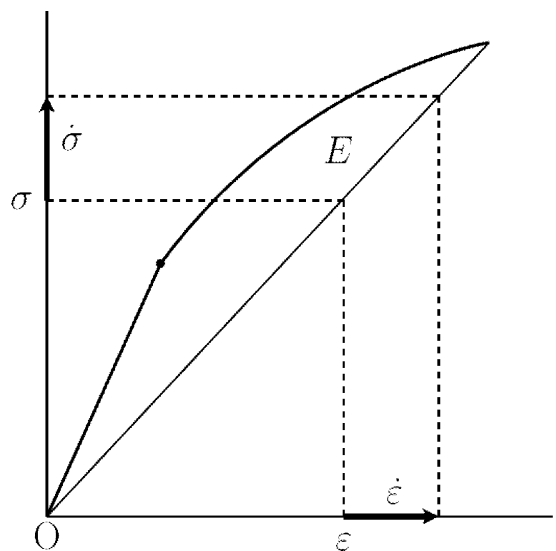

(a)

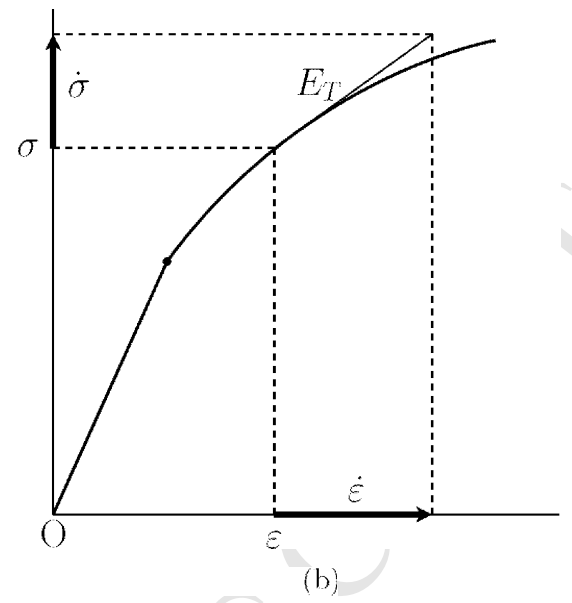

Figure 3: Stress rate response given by the $1 D$ model for a fixed temperature: (a) in the linear range, (b) for an irreversible loading.

Proof. At the time $t$, there are three cases to consider.

(i) If $\sigma<h$, then $\dot{\lambda}=0$ according to (14d). On the one hand, one derives from (14c) that $\dot{\varepsilon}^{D}=0$, and therefore that $\dot{\varepsilon}=C \dot{\sigma}$ owing to (14a), that is to say $\dot{\sigma}=E \dot{\varepsilon}$. On the other hand, one has $\dot{\alpha}=0$ thanks to (14c) and to the fact that $A \neq 0$ according to hypotheses (4)(5).

(ii) If $\sigma=h$, it follows $\dot{\sigma} \leq \dot{h}$ (using the condition of admissibility satisfied by $\sigma$ at the later times $t+\Delta t$ such that $\Delta t>0$ ). Let us first consider the case where $\dot{\sigma}<\dot{h}$. At times $t+\Delta t$ with $\Delta t>0$, we will have $\varphi<0$ and therefore (as in the case (i)):

$$
\dot{\sigma}=E \dot{\varepsilon} \quad \text { and } \quad \dot{\alpha}=0 \quad \text { at } t+\Delta t .
$$

By doing $\Delta t \rightarrow 0$, the same holds true at the time $t$ (see Remark 1).

(iii) The case $\sigma=h$ and $\dot{\sigma}=\dot{h}$ remains to examine. The multiplier $\dot{\lambda}$ can 
be eliminated between the two components of the vector equality (14c):

$$
\dot{\varepsilon}^{D}=C_{\alpha}^{\prime} \sigma \dot{\alpha}
$$

It follows that

$$
\dot{\varepsilon}=C \dot{h}+C_{\alpha}^{\prime} h \dot{\alpha}
$$

by using (14a), then

$$
\dot{\varepsilon}=\frac{d}{d t}(C h)
$$

We conclude that $\dot{\alpha}=\dot{\varepsilon}$, thanks to identity (6). Finally, we have

$$
\dot{\sigma}=h_{\alpha}^{\prime} \dot{\varepsilon}
$$

That ends the proof.

Remark 2. The current value of the internal variable is linked to the strain path as it can be seen from Proposition 1. There is no a precise method for selecting an internal variable, see Lemaitre and Chaboche (1985), and the choice is guided by the type of application under study. In the present case, the objective is to define a more general model of behavior with damage taking into account the influence of the temperature on the evolution of the damage, see section 5 . So, the previous choice $\alpha$ of the internal variable is prefered for instance to a definition linked to the elasticity modulus $E$. Let us also note that an analogous model was considered in Laborde and Michrafy (1991) using another internal variable and without considering the temperature. 


\section{Taking into account of the temperature}

We now consider a loading on a $C / C$ composite when the temperature is varying. In the first subsection, constitutive equations are introduced where the mechanical properties also depend on the temperature. Then, we study the consistency of the proposed model with respect to typical stress-strain curves given at different temperature values. The last subsection is devoted to some comments on our options of modelling about the thermo-mechanical coupling.

\subsection{Constitutive equations}

For large heat variations, the $C / C$ composite mechanical properties depend not only on damage, but also on the current temperature value (elastic stiffness, stress threshold, possible residual strains). Indeed, stress-strain experimental curves show the nonlinear dependance of the elastic stiffness with respect to the temperature, see section 8. Consequently, the current state of damage itself depends on the history of the thermal loading. From the modelling procedure introduced in the previous sections, we now propose an attempt of damage-temperature coupling which is compatible with available experimental data. The presentation is carried out within a unidimensional framework before to consider the three-dimensional case in section 6 . The residual strains are still neglected, see Remark 4 below.

The experimental basis of modelling is represented by stress-strain curves with loading-unloading cycles to a fixed temperature $\theta$, for various values of $\theta$. The stress threshold curve depends on the temperature as well as Young's modulus. Simplified stress-strain curves are shown in Figure 4 for 
two temperature values. In both cases, the mechanical loading process is considered at the fixed temperature $\left(\theta_{1}\right.$ or $\left.\theta_{2}\right)$. As previously, the Young's modulus is given by the secant stiffness.

The approach followed in section 3 can be used for each fixed value of the temperature. Let us consider the stress-strain curve relative to a mechanical loading process at the given temperature $\theta$. At each stage of the process, let us define as in section 3.1:

$$
\alpha=\frac{h}{E}-\varepsilon^{0}
$$

from the current values of the elastic stiffness $E$, the yield limit $h$ and from the initial limit of elasticity for strains $\varepsilon^{0}$ at the given temperature. As previously, we can express $E$ and $h$ as a function of $\alpha$. Here, these quantities depend on the temperature:

$$
E=E(\alpha, \theta), \quad h=h(\alpha, \theta)
$$

and $\varepsilon^{0}=\varepsilon^{0}(\theta)$.

From the definition (15), we deduce the two identities

$$
\frac{\partial}{\partial \alpha}\left(\frac{h}{E}-\varepsilon^{0}\right)=1, \quad \frac{\partial}{\partial \theta}\left(\frac{h}{E}-\varepsilon^{0}\right)=0 .
$$

Such a condition expresses that $\alpha$ and $\theta$ are independent variables in the definition of functions $h$ and $E$. Equalities (16) can be considered as a compatibility condition satisfied by the data $h$ and $E$ as a function of $\alpha$ and $\theta$. Conversely, the assumption (16) implies straightforwardly that

$$
\dot{\alpha}=\frac{d}{d t}\left(\frac{h}{E}-\varepsilon^{0}\right) .
$$




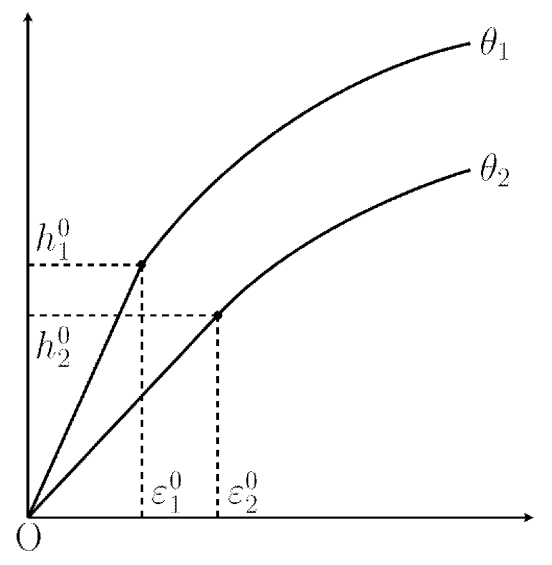

(a)

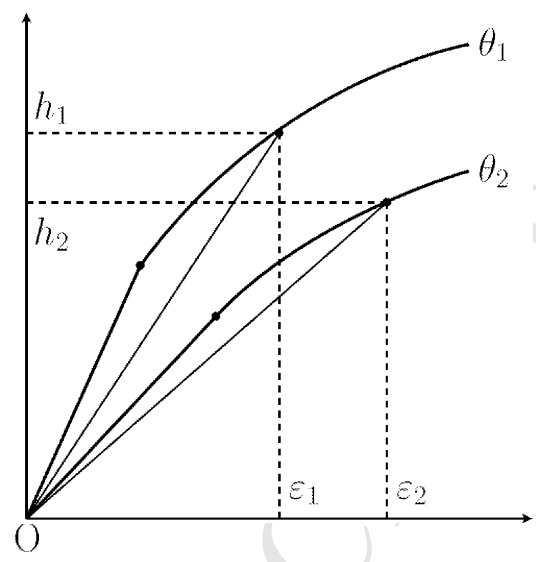

(b)

Figure 4: Behavior under a mechanical loading at fixed temperature values $\theta_{1}$ and $\theta_{2}$ : (a) initial thresholds, (b) secant moduli.

According to the approach followed in section 3.2, the admissibility criterion on the stress $\sigma$ is written as follows:

$$
\varphi(\sigma, \alpha, \theta)=\sigma-h(\alpha, \theta) \leq 0 .
$$

In the uniaxial Hooke's law, the elastic modulus is temperature-dependent now:

$$
\sigma=E(\alpha, \theta) \varepsilon .
$$

where $\varepsilon$ represents the difference between the total strain and the thermal strain, see section 6.2. The relation (19) can be written equivalently

$$
\varepsilon=C(\alpha, \theta) \sigma .
$$

Similarly to the case of a fixed temperature, the nonnegative and differentiable functions $E(\alpha, \theta), h(\alpha, \theta)$ satisfy the following conditions :

$$
E_{\alpha}^{\prime}<0, \quad h_{\alpha}^{\prime}>0
$$


and $h$ is still assumed be a concave function of the variable $\alpha$.

We can write, similarly to (14):

$$
\begin{aligned}
& \dot{\varepsilon}=C \dot{\sigma}+\dot{\varepsilon}^{D}+B \dot{\theta} \\
& \left(\dot{\varepsilon}^{D},-A \dot{\alpha}\right)=\dot{\lambda}\left(\frac{\partial \varphi}{\partial \sigma}, \frac{\partial \varphi}{\partial \alpha}\right) \\
& \varphi \leq 0, \quad \dot{\lambda} \geq 0, \quad \dot{\lambda}=0 \quad \text { if } \quad \varphi<0
\end{aligned}
$$

by denoting $B=C_{\theta}^{\prime} \sigma$ and where $A=C_{\alpha}^{\prime} h h_{\alpha}^{\prime}$ is obtained as in (13). Quantities $A, B$ and $C$ depend on $\theta$. Relationships (22) represent a unidimensional model of damage in the presence of a variable temperature.

\subsection{Analysis of the model}

Following the approach considered for a fixed temperature, let us now examine the rate principle associated to the damage model (22) in relation to a variation of temperature.

Proposition 2. Let $\sigma, \varepsilon, \alpha$ and a temperature value $\theta$ be given such that $0<\sigma \leq h(\alpha, \theta)$. Under the hypotheses of the section 5.1, the stress rate $\dot{\sigma}$ and the internal variable rate $\dot{\alpha}$ can be expressed as a function of the strain and temperature rates $\dot{\varepsilon}$ and $\dot{\theta}$ according to the following conditions.

(i) If $\sigma<h$, then $\dot{\alpha}=0$ and $\dot{\sigma}=E \dot{\varepsilon}+E_{\theta}^{\prime} \varepsilon \dot{\theta}$ (reversible range)

(ii) If $\sigma=h$, then $\dot{\alpha}=\left(\dot{\varepsilon}-\dot{\varepsilon}^{0}\right)^{+}$and, moreover

- either $\dot{\alpha}=0$ then $\dot{\sigma}=E \dot{\varepsilon}+E_{\theta}^{\prime} \varepsilon \dot{\theta}$ (unloading)

- or $\dot{\alpha}>0$ then $\dot{\sigma}=E_{T} \dot{\varepsilon}+F \dot{\theta}$ (damage range), with the notations $E_{T}=h_{\alpha}^{\prime}$ and $F=h_{\theta}^{\prime}-h_{\alpha}^{\prime}\left(\varepsilon^{0}\right)_{\theta}^{\prime}$. 
Let us observe in particular that the the evolution of the internal variable obeys the condition

$$
\dot{\alpha} \geq 0
$$

from the previous statement.

Proof. (i) If $\sigma<h$, we derive from (22) that $\dot{\lambda}=0$, so $\dot{\alpha}=0$ from the hypothesis (21), then $\dot{\varepsilon}=C \dot{\sigma}+C_{\theta}^{\prime} \sigma \dot{\theta}$. By inverting the latter equation, one obtains

$$
\dot{\sigma}=E \dot{\varepsilon}+\frac{E_{\theta}^{\prime}}{E} \sigma \dot{\theta}
$$

that is to say

$$
\dot{\sigma}=E \dot{\varepsilon}+E_{\theta}^{\prime} \varepsilon \dot{\theta}
$$

(ii) When $\sigma=h$ and $\dot{\sigma}<\dot{h}$, we find again $\dot{\alpha}=0$. Moreover, equation (24) is still true by using the same argument as in the study without temperature, see the proof of Proposition 1, point (ii). Besides, the equation $\dot{\varepsilon}=C \dot{\sigma}+C_{\theta}^{\prime} \sigma \dot{\theta}$ implies the following inequality

$$
\dot{\varepsilon} \leq C \dot{h}+C_{\theta}^{\prime} h \dot{\theta}
$$

from the conditions defining the present case and owing to the assumptions (21). In other words, one has:

$$
\dot{\varepsilon} \leq \frac{d}{d t}(C h),
$$

since $\dot{\alpha}=0$. It follows that $\dot{\varepsilon} \leq \dot{\varepsilon}^{0}+\dot{\alpha}$ from the expression (17) of $\dot{\alpha}$. We obtain finally

$$
\dot{\varepsilon} \leq \dot{\varepsilon}^{0}
$$


(iii) In the last case $\sigma=h$ and $\dot{\sigma}=\dot{h}$, by eliminating the multiplier $\dot{\lambda}$ in the relation $(22 \mathrm{~b})$, we find for a variable temperature

$$
\dot{\varepsilon}=C \dot{h}+C_{\alpha}^{\prime} h \dot{\alpha}+C_{\theta}^{\prime} h \dot{\theta},
$$

i.e. $\dot{\varepsilon}=\frac{d}{d t}(C h)$. Therefore, one obtains

$$
\dot{\varepsilon}=\dot{\alpha}+\dot{\varepsilon}^{0},
$$

and the condition $\dot{\sigma}=\dot{h}$ gives:

$$
\dot{\sigma}=h_{\alpha}^{\prime} \dot{\varepsilon}-h_{\alpha}^{\prime} \dot{\varepsilon}^{0}+h_{\theta}^{\prime} \dot{\theta}
$$

Moreover, let us note that $\dot{\alpha} \geq 0$ from the constitutive equations (22) and the hypotheses (21), so the relation (25) leads to

$$
\dot{\varepsilon} \geq \dot{\varepsilon}^{0} \text {. }
$$

By gathering the cases (ii) and (iii), we conclude that

$$
\dot{\alpha}=\left(\dot{\varepsilon}-\dot{\varepsilon}^{0}\right)^{+},
$$

which completes the proof.

The rate principle formulated in Proposition 2 for a variable temperature leads to

$$
\frac{d \sigma}{d t}=\frac{d}{d t}(E(\alpha, \theta) \varepsilon)
$$

at each time. This equality can be derived of the compatibility condition (16). In other words, the rate of residual strains is equal to zero as postulated in section 3.1 and the constitutive relations (22) define an elastic damage model. 


\subsection{Comments}

In the course of a given thermo-mechanical loading, damage and temperature are coupled together in the evolution of the elastic stiffness. The previous modelling induces a definite type of dependence of the damage process on the temperature loading. Actually, Proposition 2 can be illustrated by examinating the response given by the model when the temperature varies from some value $\theta_{1}$ to other one $\theta_{2}$ and the strain remains fixed.

The first case is defined in Figure 5 where the initial maximum elastic strains are equal to the same value $\varepsilon^{0}$ for both given temperatures $\theta_{1}$ and $\theta_{2}$. The starting stage $\left(\varepsilon, \sigma_{1}\right)$ is represented by the point $A$ on the stress-strain curve at temperature $\theta_{1}$, i.e. the stress is on the current damage threshold. When the temperature comes into $\theta_{2}$ from $\theta_{1}$ for a given strain value $\varepsilon$, we have $\dot{\alpha}=0$ according to Proposition 2 and the internal variable remains equal to $\alpha=\varepsilon-\varepsilon^{0}$ at the final stage $\left(\varepsilon, \sigma_{2}\right)$. The latter corresponds to the point $B$ on the curve at the temperature $\theta_{2}$ in Figure 5. So the stress $\sigma_{2}$ is on the damage threshold corresponding to the new temperature.

For the same data, let us now consider a first state of the system $\left(\varepsilon, \sigma_{1}\right)$ strictly below the stress-strain curve at temperature $\theta_{1}$. Represented by the point $B$ in Figure 6, this state was preliminarily obtained by carrying out an unloading step from the state $\left(\varepsilon_{A}, \sigma_{A}\right)$ on the loading curve at the temperature $\theta_{1}$. At point $B$, the internal variable and the secant stiffness therefore are equal resp. to

$$
\alpha=\varepsilon_{A}-\varepsilon^{0} \text { and } E_{1}=E\left(\alpha, \theta_{1}\right),
$$

moreover the stress is such that $\sigma_{1}=E_{1} \varepsilon<h\left(\alpha, \theta_{1}\right)$. When the temperature varies from $\theta_{1}$ to $\theta_{2}$ at the constant strain value $\varepsilon$, the response of the model 
again must verify $\dot{\alpha}=0$. The final state $\left(\varepsilon, \sigma_{2}\right)$ is represented in Figure 6 by the point $C$ without any variation of the internal variable. Then, the elastic stiffness is equal to $E_{2}=E\left(\alpha, \theta_{2}\right)$ and

$$
\sigma_{2}=E_{2} \varepsilon<h\left(\alpha, \theta_{2}\right) .
$$

The stress remains strictly below the limit of elasticity corresponding to the final temperature.

The third case is presented in Figure 7 where the initial maximum elastic strains $\varepsilon_{1}^{0}$ and $\varepsilon_{2}^{0}$ are distinct. The initial stage $A\left(\varepsilon, \sigma_{1}\right)$ corresponds to the damage value $\alpha=\varepsilon-\varepsilon_{1}^{0}$ obtained by a monotonous loading at temperature $\theta_{1}$ from the non-damaged state $O$ as indicated in Figure 7. As previously, the strain is fixed at the value $\varepsilon$ and the temperature comes into $\theta_{2}$ from $\theta_{1}$. According to the Proposition 2, we have $\dot{\alpha}=\left(-\dot{\varepsilon}^{0}\right)^{+}=0$ and the model predicts the state $\left(\varepsilon, \sigma_{2}\right)$ associated with the point $B$ such that

$$
\sigma_{2}=E_{2} \varepsilon, \quad E_{2}=E\left(\alpha, \theta_{2}\right) .
$$

Finally, in Figure 8, we consider a starting point $A\left(\varepsilon, \sigma_{1}\right)$ at the temperature $\theta_{1}$ for which the damage value is zero. At the temperature $\theta_{2}$ and for a constant strain $\varepsilon$, the damage at the final stage $B$ is $\alpha_{2}=\varepsilon-\varepsilon_{2}^{0}$ and we have here $\dot{\alpha}=\left(-\dot{\varepsilon}^{0}\right)^{+}>0$.

Anyway the model selects the response which minimizes the evolution of the internal variable of damage $\alpha$ among the admissible responses to a given thermo-mechanical loading. When the temperature varies continuously, this type of behavior remains to be validated experimentally.

In this unidimensional study, the chosen internal variable allows us to take into account the given typical stress-strain curves relative to a tensile 


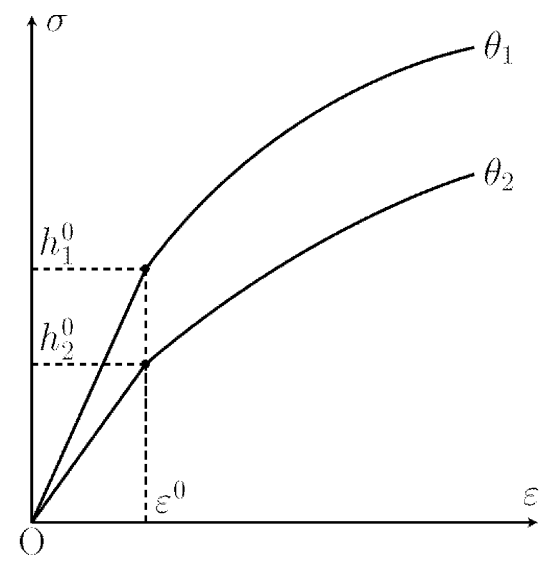

(a)

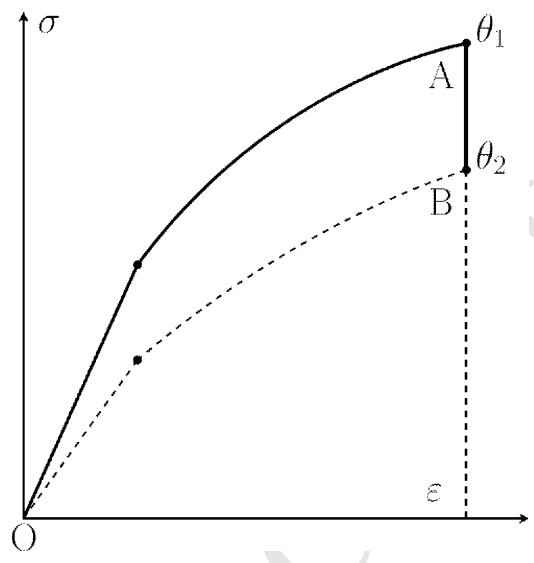

(b)

Figure 5: Variation of the temperature from $\theta_{1}$ to $\theta_{2}$ : (a) same strain threshold $\varepsilon^{0}$ at $\theta_{1}$ and $\theta_{2},(b)$ stress response from $\mathrm{A}$ to $\mathrm{B}$ for a given strain state $\varepsilon$ and for a variation of temperature from $\theta_{1}$ to $\theta_{2}$.

test at various temperatures. Uniaxial compression-tension cycles can be represented in the same way by introducing a new internal variable in compression, see section 6.2 .

\section{Three-dimensional modelling}

After reformulating the constitutive equations coming from the unidimensional analysis, we will derive a three-dimensional model generalizing the previous approach.

\subsection{Uniaxial constitutive inequality}

At a fixed temperature $\theta$, the admissibility condition (18) allows us to define the set $K(\theta)$ of the admissible pairs $(\sigma, \alpha)$ :

$$
K(\theta)=\{(\tau, \beta): \varphi(\tau, \beta, \theta) \leq 0\} .
$$




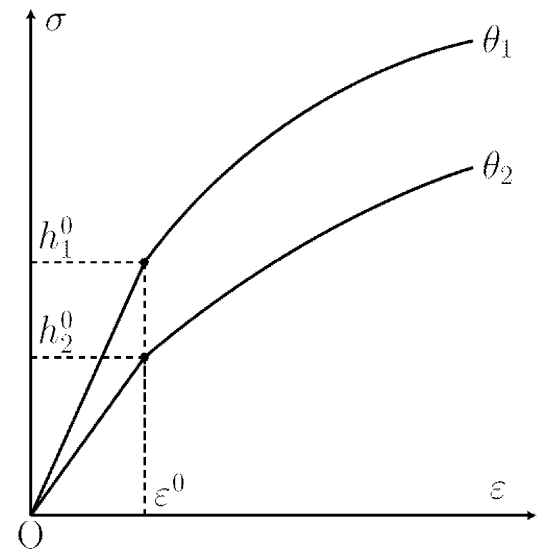

(a)

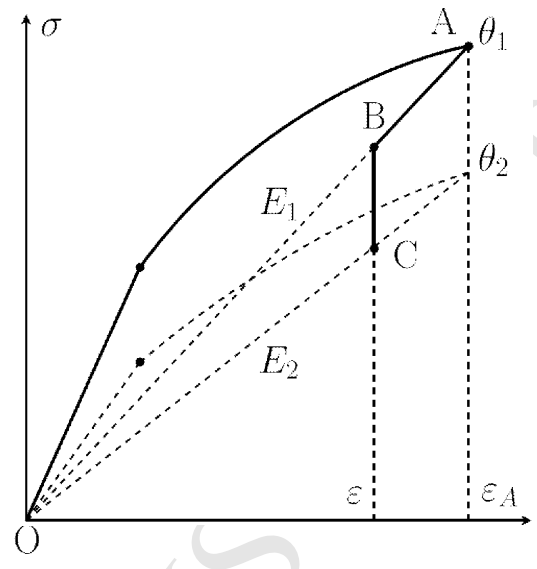

(b)

Figure 6: Temperature variation in the elastic range: (a) the loading curves at $\theta_{1}$ and $\theta_{2}$, (b) from the state $\mathrm{A}$ to $\mathrm{B}$ at temperature $\theta_{1}$, then to the state $\mathrm{C}$ at the fixed strain $\varepsilon$.

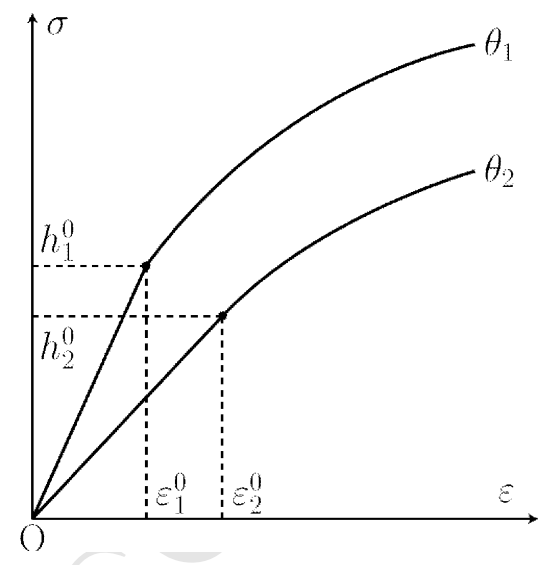

(a)

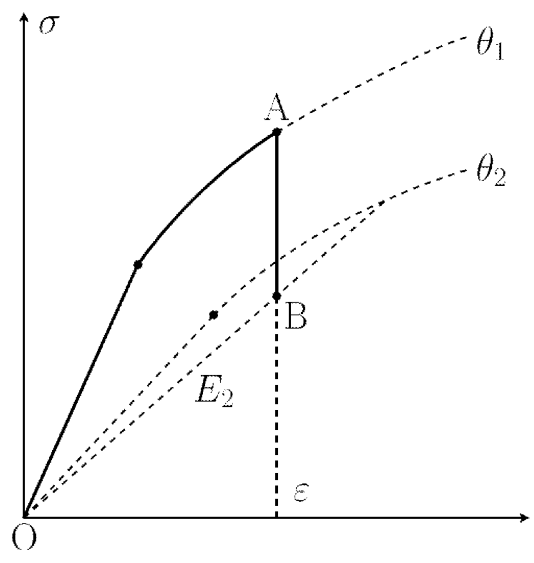

(b)

Figure 7: (a) Initial strain thresholds $\varepsilon_{1}^{0}$ and $\varepsilon_{2}^{0}$ at temperatures $\theta_{1}$ and $\theta_{2}$ respectively, (b) loading path $\mathrm{OAB}$ where $\mathrm{A}$ represents a damaged state at $\theta_{1}$. 


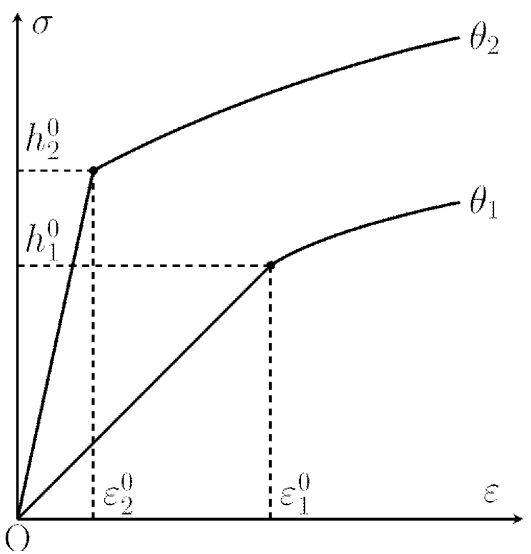

(a)

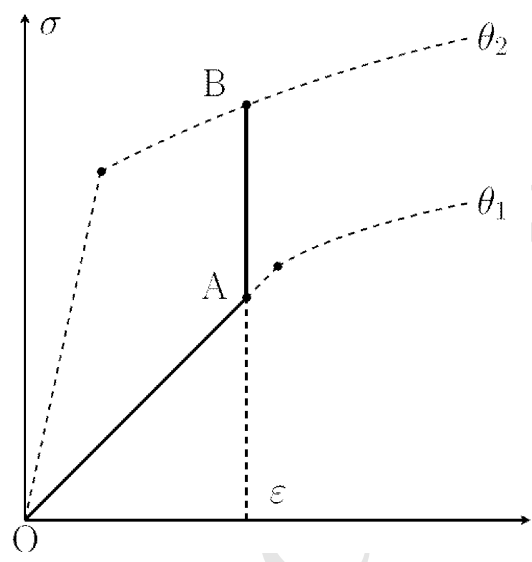

(b)

Figure 8: (a) Initial strain thresholds $\varepsilon_{1}^{0}$ and $\varepsilon_{2}^{0}$ at temperatures $\theta_{1}$ and $\theta_{2}$ respectively, (b) loading path $\mathrm{OAB}$ where $\mathrm{A}$ represents a non-damaged state at $\theta_{1}$.

The stress threshold is a concave function with respect to $\alpha$ according to (21), it follows that $K(\theta)$ is a convex set.

Constitutive relations (22) are equivalent to the following property

$$
\left(\dot{\varepsilon}^{D},-A \dot{\alpha}\right) \text { normal to } K(\theta) \text { at }(\sigma, \alpha)
$$

in the sense of Convex Analysis, that is to say

$$
\begin{aligned}
& \dot{\varepsilon}^{D}(\tau-\sigma)-A \dot{\alpha}(\beta-\alpha) \leq 0 \\
& \text { for any }(\tau, \beta) \in K(\theta), \quad \text { and }(\sigma, \alpha) \in K(\theta) .
\end{aligned}
$$

In other words, relationships (22) are equivalent to the following stressstrain relationship of inequality type

$$
\begin{aligned}
& C \dot{\sigma}(\tau-\sigma)+A \dot{\alpha}(\beta-\alpha) \geq(\dot{\varepsilon}+H \dot{\theta})(\tau-\sigma) \\
& \text { for any }(\tau, \beta) \in K(\theta), \quad \text { and }(\sigma, \alpha) \in K(\theta),
\end{aligned}
$$

with the notations of section 5.1 and $H=-B$. Such an expression of the 
unidemensional model links the strain rate to the stress and internal variable rates via the variation of temperature.

\subsection{The general modelling framework}

In this section, we propose a general expression of the behavior of a $C / C$ composite subjected to a thermo-mechanical loading in the framework of infinitesimal strains and displacements. In order to take into accont the orthotropic symmetry of the material, the reference axes in the threedimensional space $\mathbb{R}^{3}$ are defined as the three directions of fibers. The strain tensor $\varepsilon=\left\{\varepsilon_{i j}\right\}$ and the stress tensor $\sigma=\left\{\sigma_{i j}\right\}$ lie in the space $\mathbb{S}$ of the symmetrical second order tensors, equipped with the usual inner product $\sigma: \varepsilon=\sum_{i j} \sigma_{i j} \varepsilon_{i j}$. The tensor $\varepsilon$ will represent the difference between the total strains and the thermal strains, see Remark 3. The variable of damage $\alpha$ will be defined from a number of scalar or tensorial unknown quantities assigned to some irreversible properties of the material according to the fiber directions as it will be illustrated later.

The previous unidimensional analysis led to inequality (27) which can be generalized in the three-dimensional case as follows

$$
\begin{aligned}
& C \dot{\sigma}:(\tau-\sigma)+A \dot{\alpha} \cdot(\beta-\alpha) \\
& \quad \geq(\dot{\varepsilon}+H \dot{\theta}):(\tau-\sigma) \\
& \text { for any }(\tau, \beta) \in K(\theta), \quad \text { and }(\sigma, \alpha) \in K(\theta) .
\end{aligned}
$$

We have denoted $\alpha \cdot \beta$ the inner product defined in the internal variable space. The four order symmetrical tensor $C=C(\alpha, \theta)$ of the elastic compliances is a function of the damage state and is temperature dependent. It can also depend on the stress state $\sigma$ in order to take into account the difference of 
behavior in tension or in compression. The evolution of the internal variable is governed by a linear operator $A=A(\sigma, \alpha, \theta)$ also depending on $(\alpha, \theta)$ and possibly on $\sigma$ from the internal variable space into itself. Moreover. $H=H(\sigma, \alpha, \theta)$ stands for a second order symmetrical tensor concerning the involvement of the temperature variation in the mechanical behavior. Finally, $K(\theta)$ is the convex set of the admissible pairs $(\sigma, \alpha)$ at the temperature $\theta$.

For the sake of simplicity, residual strains are neglected in the damage model under study, see Remark 4 below. An extension of the mathematical framework (28) is being examined to take into account residual strains. Naturally, the identification of the flow rule of damage with residual strains will need convenient experimental informations.

Let us also note that the rate inequality (28) can also be written in the form of equalities analogous to (22) involving many multipliers. Finally, the constitutive equations (28) define a general three-dimensional model of irreversible behavior for damage problems in the presence of a temperature variation.

Remark 3. Thermal strains $\varepsilon^{\text {th }}$ do not appear explicitly in the model (28). The difference between the total strain and the mechanical strain can be written for each orthotropic direction $i$ :

$$
\varepsilon_{i j}^{t h}=\kappa_{i}(\theta)\left(\theta-\theta_{R}\right) \delta_{i j}
$$

where $\kappa_{i}(\theta)$ is the thermal dilatation coefficient of the material, $\theta_{R}$ a reference temperature and $\delta=\left\{\delta_{i j}\right\}$ denotes the Kronecker tensor. The thermal dilatation coefficient is determined by appropriate tests. In our problem, the material damage due to mechanical loadings does not significantly modify the experimental value of this coefficient. Actually, thermal strains are 
taken into account in (28) by considering that the notation $\varepsilon$ stands for the difference between the total strain and the thermal strain.

\subsection{An example}

This framework of modelling can be particularized according to the available data in order to obtain identifiable specific constitutive relationships from the given experimental loading-unloading curves. For instance, let us consider an orthotropic behavior where no account is taken of :

- the directional coupling of damage (e.g. the elastic stiffness into a fiber direction is not affected by a tensile load into another fiber direction),

- the possible presence of residual strains.

Experimental tests on $C / C$ composites showed that:

- Poisson's ratios are negligible,

- in fiber direction $i$, the Young's modulus $E_{i}$ is different in tension or in compression $(i=1,2,3)$,

- the shear moduli $G_{i j}$ are strongly dependent on the damage $(i \neq j)$, see Remark 4.

The Young's modulus $E_{i}$ in tension $\left(\sigma_{i i} \geq 0\right)$ will be denoted $E_{i}^{+}$; in compression $\left(\sigma_{i i}<0\right)$, we will write $E_{i}=E_{i}^{-}$. Similarly for the shear modulus, we will have $G_{i j}=G_{i j}^{+}$if $\sigma_{i j} \geq 0, G_{i j}=G_{i j}^{-}$otherwise. The matrix of compliances $C=C(\alpha, \theta)$ satisfies $C \sigma=\varepsilon$ with

$$
\varepsilon_{i i}=\frac{1}{E_{i}} \sigma_{i i} \text { and } \varepsilon_{i j}=\frac{1}{2 G_{i j}} \sigma_{i j}(i \neq j) .
$$

Let $\alpha$ be the internal variable lying in $\mathbb{R}^{12}$ :

$$
\alpha=\left\{\alpha_{i j}^{+}, \alpha_{i j}^{-}: i \leq j\right\}
$$


such that we have

$$
\begin{array}{ll}
E_{i}^{+}=E_{i}^{+}\left(\alpha_{i i}^{+}, \theta\right), & E_{i}^{-}=E_{i}^{-}\left(\alpha_{i i}^{-}, \theta\right), \\
G_{i j}^{+}=G_{i j}^{+}\left(\alpha_{i j}^{+}, \theta\right), & G_{i j}^{-}=G_{i j}^{-}\left(\alpha_{i j}^{-}, \theta\right) .
\end{array}
$$

The admissibility criterion associated to a uniaxial tensile stress into the fiber direction $i$ for a tension or compression loading is written:

$$
\varphi_{i i}^{+}=\sigma_{i i}-h_{i i}^{+} \leq 0 \quad \text { or } \quad \varphi_{i i}^{-}=-\sigma_{i i}-h_{i i}^{-} \leq 0
$$

where

$$
h_{i i}^{+}=h_{i i}^{+}\left(\alpha_{i i}^{+}, \theta\right) \geq 0 \quad \text { and } \quad h_{i i}^{-}=h_{i i}^{-}\left(\alpha_{i i}^{-}, \theta\right) \geq 0
$$

are resp. the tension or compression stress threshold into direction $i$. Similarly, the criterion associated with the shear test in the plane $(i, j)$ is defined as:

$$
\varphi_{i j}^{+}=\sigma_{i j}-h_{i j}^{+} \leq 0 \quad \text { and } \quad \varphi_{i j}^{-}=-\sigma_{i j}-h_{i j}^{-} \leq 0,
$$

denoting

$$
\begin{aligned}
& h_{i j}^{+}=h_{i j}^{+}\left(\alpha_{i j}^{+}, \theta\right) \geq 0 \quad \text { if } \sigma_{i j} \geq 0, \\
& h_{i j}^{-}=h_{i j}^{-}\left(\alpha_{i j}^{-}, \theta\right) \geq 0 \quad \text { otherwise. }
\end{aligned}
$$

The global admissibility condition at the temperature $\theta$ is finally written:

$$
\varphi(\sigma, \alpha, \theta) \leq 0
$$

with

$$
\varphi=\max \left\{\varphi_{i j}^{+}, \varphi_{i j}^{-}: i \leq j\right\} .
$$


In the present particularization of the model (28), $A=A(\alpha, \theta)$ is a diagonal matrix. Refering to relations (22) obtained in the unidimensional analysis, the first diagonal terms take the following form:

$$
\left(\frac{\partial}{\partial \alpha_{i i}} \frac{1}{E_{i}}\right) h_{i i}\left(\frac{\partial}{\partial \alpha_{i i}} h_{i i}\right) \text {. }
$$

In this expression as in the following ones, the quantities $E_{i}, h_{i}$ and $\alpha_{i}$ are indexed by + or - depending on the sign of $\sigma_{i i}$. In the same way, the last three diagonal terms of $A$ are written:

$$
\left(\frac{\partial}{\partial \alpha_{i j}} \frac{1}{2 G_{i j}}\right) h_{i j}\left(\frac{\partial}{\partial \alpha_{i j}} h_{i j}\right)
$$

The elastic moduli are assumed be differentiable, nonnegative and decreasing functions with respect to their corresponding internal variable :

$$
\frac{\partial E_{i}}{\partial \alpha_{i i}}<0, \quad \frac{\partial G_{i j}}{\partial \alpha_{i j}}<0
$$

by analogy with (21) for the Young modulus in the unidimensional case. In the same way, the threshold $h_{i j}$ is a differentiable, nonnegative and increasing function of $\alpha_{i j}$ (compare to (21)) and we also suppose the concavity of $h_{i j}$ with respect to $\alpha_{i j}$.

Under the above assumptions, the thermodynamic consistency of the simplified model of damage is ensured if the evolution of the internal variables satisfies :

$$
\dot{\alpha}_{i j} \geq 0
$$

from AppendixA. The property (30) derives from the application of Proposition 2 to the uncoupled model under study, see inequality (23).

We have considered a directional uncoupling of the damage for the lack of corresponding physical data. A first improvement is to consider a Young's 
modulus into the direction of fiber 1 as follows

$$
E_{1}=E\left(\alpha_{11}^{*}, \theta\right)
$$

where $\alpha_{11}^{*}$ represents an equivalent damage:

$$
\alpha_{11}^{*}=\alpha_{11}+a \alpha_{22}+b \alpha_{33} .
$$

Phenomenological constants $a$ and $b$ measure the influence of directions 2 and 3 on direction 1 . According the available data, the proposed modelling procedure can be used for more complex directional coupling effects.

The general framework (28) offers a flexibility of the modelling which allows to consider more complex situations than in the present specific application. That depends on the available experimental loading-unloading curves.

Remark 4. Beyond the initial stress threshold of damage, characteristic loading tests show that the shear moduli of the material are highly variable with respect to the applied loading beyond the initial stress threshold of damage. Macroscopic cracks appear in the matrix during the loading and the strands do not bring the material back to its original state at unloading. So residual strains are not negligible in shear planes, contrary to tension and compression tests. No residual strain is considered in the damage model with temperature studied in this subsection. But the knowledge of loadingunloading curves in the shear planes makes possible to take into account important residual strains in an extension of the model. 


\section{The incremental scheme}

Let us see how the previous modelling can be be exploited from a computational viewpoint. The model of damage defines the constitutive equations to be combined with the equations of dynamics and the force/displacement boundary conditions. We are interested in an implicit time discretization of the finite element problem. Classically, in order to update the nodal force vector in the (global) incremental equilibrium problem, the nonlinear constitutive equations are treated locally (at each integration point). In this section, we study a local (pointwise) implict incremental scheme to approximate the model of damage.

We have postulated a directional uncoupling of damage for the orthotropic materials under consideration. So the discretization method for the threedimensional constitutive equations at a given point is reduced to a unidimensional approximation problem in each fiber direction. We now consider things in the unidimensional framework presented in section 6.1.

Let $t_{n}$ and $t_{n+1}$ be two consecutive discrete times of the loading path in tension (i.e. $\sigma_{n}>0, \sigma_{n+1}>0$ ) and $\Delta t=t_{n+1}-t_{n}>0$. The discrete value of a quantity $q$ at $t_{n}$ is denoted $q_{n}$ and its increment between $t_{n}$ and $t_{n+1}$ is written $\Delta q=q_{n+1}-q_{n}$. Moreover, if the quantity $q$ depends on $\alpha$ and $\theta$, we will use the convention:

$$
q_{n+1 / 2}=q\left(\alpha_{n}, \theta_{n+1}\right)
$$

The initial data satisfy the conditions

$$
\varepsilon_{0}=C\left(\alpha_{0}, \theta_{0}\right) \sigma_{0}, \quad \sigma_{0} \leq h\left(\alpha_{0}, \theta_{0}\right) .
$$


The step by step scheme is formulated as follows at the discrete time $\left(t_{n+1}\right)$. Let $\varepsilon_{n+1}$ be given, the trial stress $\sigma^{T}$ is defined by the relation

$$
C_{n+1 / 2} \sigma^{T^{\prime}}=\varepsilon_{n+1}
$$

and $\varepsilon_{n}, \sigma_{n}$ as well as $\alpha_{n}$ are known at this stage such that $\varepsilon_{n}=(C \sigma)_{n}$. Then, $\sigma_{n+1}$ and $\alpha_{n+1}$ are computed from $\sigma^{T}$ according to the following two cases (Figure 9).

$$
\begin{gathered}
\text { If } \sigma^{T^{\prime}} \leq h_{n+1 / 2}, \text { then } \\
\alpha_{n+1}=\alpha_{n}, \sigma_{n+1}=\sigma^{T} . \\
\text { If } \sigma^{T^{\prime}}>h_{n+1 / 2}, \text { then } \\
\alpha_{n+1}=\left(\varepsilon-\varepsilon^{0}\right)_{n+1}, \sigma_{n+1}=h_{n+1} .
\end{gathered}
$$

One can verify that the projection type algorithm (32) implies

$$
\varepsilon_{n+1}=(C \sigma)_{n+1}
$$

at each step (compare to (20)) and, moreover

$$
\alpha_{n+1} \geq \alpha_{n} .
$$

The following statement shows how the algorithm is connected to the damage model.

Proposition 3. The step by step method (32) is equivalent to the following 


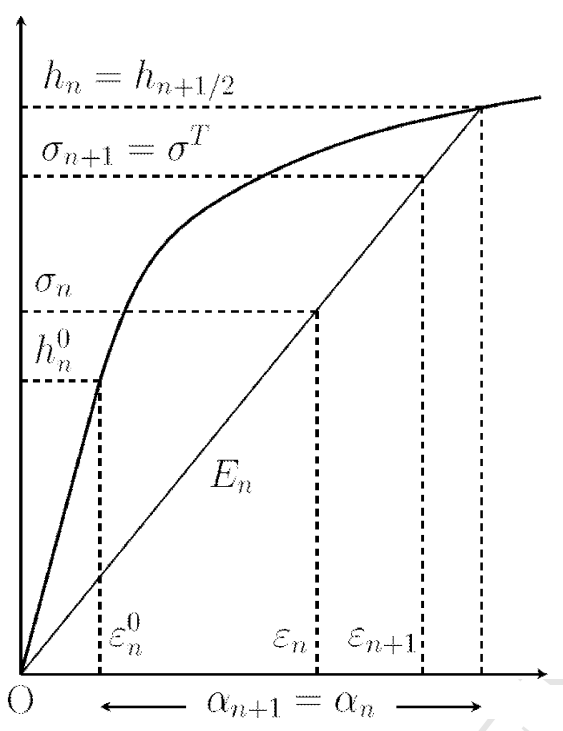

(a)

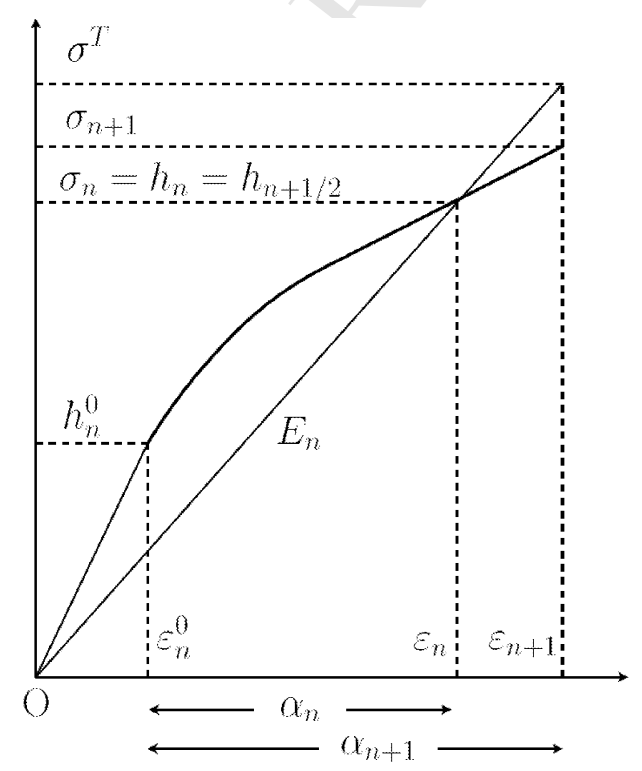

(b)

Figure 9: The incremental scheme for $\theta_{n+1}=\theta_{n}$ : (a) when $\sigma^{T} \leq h_{n+1 / 2}$, (b) when $\sigma^{T}>h_{n+1 / 2}$. 
variational inequality relative to the unknowns $\left(\sigma_{n+1}, \alpha_{n+1}\right)$

$$
\begin{aligned}
& C_{n+1 / 2} \Delta \sigma\left(\tau-\sigma_{n+1}\right) \\
& +\left(C_{n+1}-C_{n+1 / 2}\right)\left(h h_{\alpha}^{\prime}\right)_{n+1}\left(\beta-\alpha_{n+1}\right) \\
& \geq \Delta \varepsilon\left(\tau-\sigma_{n+1}\right) \\
& -\left(C_{n+1 / 2}-C_{n}\right) \sigma_{n}\left(\tau-\sigma_{n+1}\right) \\
& \text { for any }(\tau, \beta) \in K\left(\theta_{n+1}\right) \text {, } \\
& \text { with }\left(\sigma_{n+1}, \alpha_{n+1}\right) \in K\left(\theta_{n+1}\right) \text {. }
\end{aligned}
$$

The above variational inequality corresponds to an implicit type time discretization of the inequality (27) obtained from the constitutive relations Glowinski et al. (1976). As a matter of fact, we see in the inequality (33) that

$$
\begin{aligned}
& C_{n+1}-C_{n+1 / 2}=C_{\alpha}^{\prime}\left(\tilde{\alpha}_{n+1}, \theta_{n+1}\right) \Delta \alpha, \\
& C_{n+1 / 2}-C_{n}=C_{\theta}^{\prime}\left(\alpha_{n}, \tilde{\theta}_{n+1}\right) \Delta \theta
\end{aligned}
$$

for some intermediate values

$$
\tilde{\alpha}_{n+1} \in\left[\alpha_{n}, \alpha_{n+1}\right], \quad \tilde{\theta}_{n+1} \in\left[\theta_{n}, \theta_{n+1}\right]
$$

thanks to the mean value theorem. In that sense, the step by step scheme will be said consistent to the uniaxial damage model with variable temperature. At $t_{n+1}$, the discrete inequality (33) relies the incremental stress $\sigma_{n+1}$ and the internal variable $\alpha_{n+1}$ to the strain $\varepsilon_{n+1}$ (computed by an iterative procedure in the context of the incremental finite element problem).

PROOF. Let us first check that the algorithm is derived from the variational inequality when the temperature is fixed. 
A - The case without temperature variation.

If $\theta_{n+1}=\theta_{n}$, the discrete inequality (33) can be simplified as follows

$$
\begin{aligned}
& \begin{array}{l}
C_{n} \Delta \sigma\left(\tau-\sigma_{n+1}\right) \\
\qquad+\left(C_{n+1}-C_{n}\right)\left(h h_{\alpha}^{\prime}\right)_{n+1}\left(\beta-\alpha_{n+1}\right) \\
\quad \geq \Delta \varepsilon\left(\tau-\sigma_{n+1}\right)
\end{array} \\
& \text { for all }(\tau, \beta) \in K_{n+1}, \\
& \text { with }\left(\sigma_{n+1}, \alpha_{n+1}\right) \in K_{n+1} .
\end{aligned}
$$

Moreover, the definition (31) of the trial stress $\sigma^{T}$ becomes

$$
C_{n} \sigma^{T}=\varepsilon_{n+1}
$$

Then, the discrete inequality (34) can be written:

$$
\begin{aligned}
& C_{n}\left(\sigma_{n+1}-\sigma^{T}\right)\left(\tau-\sigma_{n+1}\right) \\
& \quad+\left(C_{n+1}-C_{n}\right)\left(h h_{\alpha}^{\prime}\right)_{n+1}\left(\beta-\alpha_{n+1}\right) \geq 0 \\
& \text { for any }(\tau, \beta) \in K_{n+1}, \\
& \text { with }\left(\sigma_{n+1}, \alpha_{n+1}\right) \in K_{n+1}
\end{aligned}
$$

by using the recurrence hypothesis $(C \sigma)_{n}=\varepsilon_{n}$. This is the formulation of a condition of normality with respect to the convex set $K_{n+1}$. So there is a multiplier $\Delta \lambda$ such that

$$
\begin{array}{r}
\left(C_{n}\left(\sigma^{T}-\sigma_{n+1}\right),-\left(C_{n+1}-C_{n}\right)\left(h h_{\alpha}^{\prime}\right)_{n+1}\right) \\
=\Delta \lambda\left(\frac{\partial \varphi}{\partial \sigma}, \frac{\partial \varphi}{\partial \alpha}\right)_{n+1} \\
\varphi_{n+1} \leq 0, \quad \Delta \lambda \geq 0, \quad \Delta \lambda \varphi_{n+1}=0
\end{array}
$$

where the gradient of $\varphi$ is equal to $\left(1,-\left(h_{\alpha}^{\prime}\right)_{n+1}\right)$. 
To derive the step by step scheme from this system of complementarity, we proceed by recurrence. At the step $n=0$, we put $C_{0} \sigma_{0}=\varepsilon_{0}$. At the step $(n+1)$, assuming that $C_{n} \sigma_{n}=\varepsilon_{n}$, there are two possibilities.

(i) Either $\varphi_{n+1}<0$, so we have $\Delta \lambda=0$ according to (37b). Then the equation (37a) on the one hand gives $\sigma_{n+1}=\sigma^{T}$ and, on the other hand $C_{n+1}=C_{n}$. The equality $E_{n+1}=E_{n}$ implies that $\alpha_{n+1}=\alpha_{n}$ because the function $E(\alpha)$ is strictly decreasing by assumption (4). Moreover, from definition (35) of $\sigma^{T}$, it follows that $(C \sigma)_{n+1}=\varepsilon_{n+1}$. Finally, we observe that $\sigma^{T} \leq h_{n}$ since $\varphi_{n+1}<0$.

(ii) Or $\varphi_{n+1}=0$, i.e. $\sigma_{n+1}=h_{n+1}$, then one derives from (37):

$$
C_{n}\left(\sigma^{T}-\sigma_{n+1}\right)=\Delta \lambda=\left(C_{n+1}-C_{n}\right) h_{n+1} .
$$

This relation leads to

$$
(C \sigma)_{n+1}=C_{n} \sigma^{T}
$$

hence $(C \sigma)_{n+1}=\varepsilon_{n+1}$ thanks to the definition (35) of $\sigma^{T}$. It follows that $\varepsilon_{n+1}=(C h)_{n+1}=\alpha_{n+1}+\varepsilon_{n+1}^{0}$ by definition of the functions $E(\alpha)$ and $h(\alpha)$, i.e.:

$$
\alpha_{n+1}=\varepsilon_{n+1}-\varepsilon_{n+1}^{0}
$$

It remains to verify that

$$
\sigma^{T} \geq h_{n}
$$

It is sufficient to consider the relation (38) and to take into account the fact that $\Delta \lambda \geq 0$ together with the assumptions of monoticity 
on functions $E$ and $h$. On the one hand, we see that $\Delta C \geq 0$, therefore $\Delta \alpha \geq 0$ and then $\Delta h \geq 0$. On the other hand, one has $\sigma^{T}-\sigma_{n+1} \geq 0$ with $\sigma_{n+1}=h_{n+1}$, hence finally (39).

$\mathrm{B}$ - The problem with a temperature variation.

At the step $(n+1)$, the trial stress $\sigma^{T^{\prime}}$ defined by (31) satisfies the following identity

$$
C_{n+1 / 2}\left(\sigma_{n+1}-\sigma^{T}\right)=C_{n+1 / 2} \Delta \sigma+\left(C_{n+1 / 2}-C_{n}\right) \sigma_{n}-\Delta \varepsilon .
$$

where we have used the recurrence hypothesis $(C \sigma)_{n}=\varepsilon_{n}$. The equality (33) is then equivalent to

$$
\begin{aligned}
& C_{n+1 / 2}\left(\sigma_{n+1}-\sigma^{T}\right)\left(\tau-\sigma_{n+1}\right) \\
& \quad+\left(C_{n+1}-C_{n+1 / 2}\right)\left(h h_{\alpha}^{\prime}\right)_{n+1}\left(\beta-\alpha_{n+1}\right) \geq 0 \\
& \text { for any }(\tau, \beta) \in K_{n+1}, \\
& \text { with }(\sigma, \alpha) \in K_{n+1} .
\end{aligned}
$$

As above, there is therefore $\Delta \lambda$ such that

$$
\begin{aligned}
\left(C_{n+1 / 2}\left(\sigma^{T^{\prime}}-\sigma_{n+1}\right),-\left(C_{n+1}-C_{n+1 / 2}\right)\right. & \left.\left(h h_{\alpha}^{\prime}\right)_{n+1}\right) \\
& =\Delta \lambda\left(\frac{\partial \varphi}{\partial \sigma}, \frac{\partial \varphi}{\partial \alpha}\right)_{n+1} .
\end{aligned}
$$

(i) If $\varphi_{n+1}<0$, then $\Delta \lambda=0$ and it results from (40) that

$$
\sigma_{n+1}=\sigma^{T} .
$$

Moreover, we have $C_{n+1}=C_{n+1 / 2}$ and therefore

$$
\alpha_{n+1}=\alpha_{n}
$$


since the quantity $C\left(\alpha, \theta_{n+1}\right)$ is strictly increasing with respect to $\alpha$ according to the assumption (21). As in the Part A, this gives

$$
\varepsilon_{n+1}=(C \sigma)_{n+1}
$$

Finally, the condition $\varphi_{n+1} \leq 0$ is written

$$
\sigma^{T} \leq h_{n+1 / 2}
$$

(ii) If $\varphi_{n+1}=0$, it results from (40)

$$
\begin{aligned}
& C_{n+1 / 2}\left(\sigma^{T}-\sigma_{n+1}\right) \\
& \quad=\Delta \lambda=\left(C_{n+1}-C_{n+1 / 2}\right) h_{n+1}
\end{aligned}
$$

whence

$$
C_{n+1 / 2} \sigma^{T}=C_{n+1} h_{n+1}
$$

since $\sigma_{n+1}=h_{n+1}$. Thanks to the definition (31) of $\sigma^{T}$, it follows that $\varepsilon_{n+1}=(C h)_{n+1}$ and

$$
\alpha_{n+1}=\varepsilon_{n+1}-\varepsilon_{n+1}^{0}
$$

see Part A. Let us now check that

$$
\sigma^{T} \geq h_{n+1 / 2}
$$

The condition $\Delta \lambda \geq 0$ together with the relation (41) ensures that, on the one hand $C_{n+1}-C_{n+1 / 2} \geq 0$, therefore $\Delta \alpha \geq 0$ and $h_{n+1}-h_{n+1 / 2} \geq 0$. On the other hand $\sigma^{T}-\sigma_{n+1} \geq 0$ and therefore $\sigma^{T} \geq h_{n+1}$, hence the assertion.

Conversely, we can prove that $\left(\sigma_{n+1}, \alpha_{n+1}\right)$ defined by the scheme (32) is solution to the variational inequality (33) by using similar arguments. 
Remark 5. The mathematical analysis of the convergence of the incremental procedure when $\Delta t \rightarrow 0$ is beyond the scope of this study.

\section{Simulations of damage in a quasi-static loading}

A first numerical example is presented in this section from the simplified orthotropic model introduced in section 6.3.

\subsection{Computation methodology}

The problem of quasi-static evolution is discretized in an implicit way owing to the projection algorithm (32). The algorithmic strategy for the finite element problem was implemented by using the commercial software ABAQUS ${ }^{\circledR}$ (in the user subroutine Umat), see Figure 10.

For the specific model of section 6.3 , the behavior of the material 3D $\mathrm{C}-\mathrm{C}$ is identified from tension, compression and shear uniaxial quasi-static loadings at different temperatures. The numerical example below is relative to a uniaxial tensile test. In Figure 11, each loading curve requires five experimental tests from which an average curve of behavior is built. The uncertainties are less than 10\%. The resulting stress-strain curves were coded in a tabular form for three values of the temperature. In the data tables obtained from the experimental curves of Figure 11, about twenty pair $(\varepsilon, \sigma)$ are given for each curve from the origin to the failure point, see Tables 1 and 2. The postfailure is not taking into account in this present work.

The initial strain thresholds are zero in the present case. Therefore the internal variable associated to each of these monotonous uniaxial loading is equal to the corresponding current state of longitudinal elongation $\varepsilon$. So the identification of the yield function and of the elastic stiffness is directly 
derived from the relations $h(\alpha, \theta)=\sigma$ and $E(\alpha, \theta)=\sigma / \varepsilon$ respectively. The sign of the uniaxial stress $\sigma$ is taking into account for evaluating the yield function and the elastic stiffness. For a computed value $\alpha$ and for a given temperature $\theta$, the value of $h$ is obtained from the tables by a double interpolation with respect to $\alpha$ and $\theta$. Then $E(\alpha, \theta)$ is derived from $h(\alpha, \theta)$. The values of the initial stiffness $E_{0}$ are experimental data given in Tables 1 and 2. The step of calibration were coded in the subroutine Umat of ABAQUS. The identification is automatic and requires no work to make input data.

\subsection{A numerical example}

Let $\Omega$ be the reference configuration of the deformable body in the threedimensional space and $u=\left(u_{i}\right)$ the displacement field of particles $x=\left(x_{i}\right)$ in $\Omega$ within the context of the infinitesimal strains theory. In the following uniaxial tension-compression test, the initial geometry is the unit cube

$$
\Omega=\left\{x=\left(x_{i}\right): 0 \leq x_{i} \leq 1\right\}
$$

subjected to the displacement boundary conditions:

$$
\begin{array}{ll}
u_{1}=0 \text { on the face } & \left\{x_{1}=0\right\}, \\
u_{2}=0 \text { on the face } & \left\{x_{2}=0\right\}, \\
u_{3}=0 \text { on the face } & \left\{x_{3}=0\right\}
\end{array}
$$

and $u_{1}$ is prescribed at each step on the face $\left\{x_{1}=1\right\}$. The complementary boundary conditions are of null force type and the body forces are neglected. The values of the material constants are directly calculated from the given experimental stress-strain curves, see section 8.1. For this uniaxial test, a 
coarse mesh of heigh-node bilinear isoparametric elements is used. The convergence tolerance in residual forces is equal to $0.5 \%$ and $1 \%$ in displacement.

The incremental thermo-mechanical loading includes eight stages presented in Figure 11. During the stage 1, a displacement of $32 \times 10^{-3} \mathrm{~m}$ is prescribed into the normal direction on the face $\left\{x_{1}=1\right\}$, whereas the temperature is fixed at $2750^{\circ} \mathrm{C}$. The displacement condition is applied in an incremental way. The final solution is independent of the value of the incrementation step. It is represented in Figure 11 by the point 1 which is clearly located on the experimental tension curve.

The stage 2 consists of maintaining the previous fixed displacement while the body is progressively cooled down to $2500^{\circ} \mathrm{C}$, with a resultant position on the corresponding experimental load curve.

During the stage 3 , the deformable solid is unloaded by an prescribed displacement $U_{1}=20 \times 10^{-3} \mathrm{~m}$ while maintaining the temperature at $2500^{\circ} \mathrm{C}$. The incremental solution is a straight line passing through the origin of the axes and the state 2 , the slope of which corresponds to the damage value at the previous stage.

During the stage 4 , the temperature is again increased to $2750^{\circ} \mathrm{C}$ with the same prescribed displacement. The state 4 is on the unloading path obtained in the first stage.

The stage 5 consists of unloading the body by a zero displacement condition again in the direction 1 at $2750^{\circ} \mathrm{C}$.

The first five stages were performed according to imposed displacements generating tension stresses. In the following three stages, a pressure is applied to the face $\left\{x_{1}=1\right\}$ of the cube, thus generating compression stresses. 
During the stage 6 , a pressure of $186 \times 10^{6} \mathrm{~Pa}$ is applied whereas the temperature goes back to $2500^{\circ} \mathrm{C}$. As the experimental data for compression at $2500{ }^{\circ} \mathrm{C}$ are not available, the final and intermediary solutions are obtained by interpolation based on the data known at $2200^{\circ} \mathrm{C}$ and $2750^{\circ} \mathrm{C}$.

The stage 7 consists of maintaining the previous pressure level and of increasing the temperature up to $2750^{\circ} \mathrm{C}$. The solution obtained is localized on the experimental compression curve performed at $2750{ }^{\circ} \mathrm{C}$.

Finally, the last stage relieves the load with the pressure progressively dropping to zero, whilst the temperature is held at $2750{ }^{\circ} \mathrm{C}$. Unloading is performed according to a straight line passing through the origin.

The results obtained confirm the fact that this numerical modelling approach is able of reproducing exactly the experimental data considered.

\section{Conclusion}

To our knowledge, this work is an original mathematical modelling attempt for the coupling of damage and temperature in thermostructural composites. This approach leads to a flexible and efficient numerical methodology for simulating the experimental behavior of $3 \mathrm{D} C / C$ composites. The model is well-adapted to a computational treatement.

\section{Acknowledgements}

This work was supported by the CEA-CESTA. The authors gratefully acknowledge Mrs Lambelin, Cosculluela and Ployart of the CEA-CESTA for their support. We also thank Mr. Tallaron of the CEA for assistance with the supply of experimental data. 
1. At increment $n$ are known: $\left\{\varepsilon_{n}, \sigma_{n}, \alpha_{n}, \theta_{n}\right\}$.

2. At increment $n+1$ are known: $\left\{\varepsilon_{n+1}, \theta_{n+1}\right\}$ hence:

$$
\Delta \varepsilon=\varepsilon_{n+1}-\varepsilon_{n} ; \quad E_{n+1 / 2}^{+}=E^{+}\left(\alpha_{n}^{+}, \theta_{n+1}\right) ; \quad E_{n+1 / 2}^{-}=E^{-}\left(\alpha_{n}^{-}, \theta_{n+1}\right) .
$$

\section{Prediction phase.}

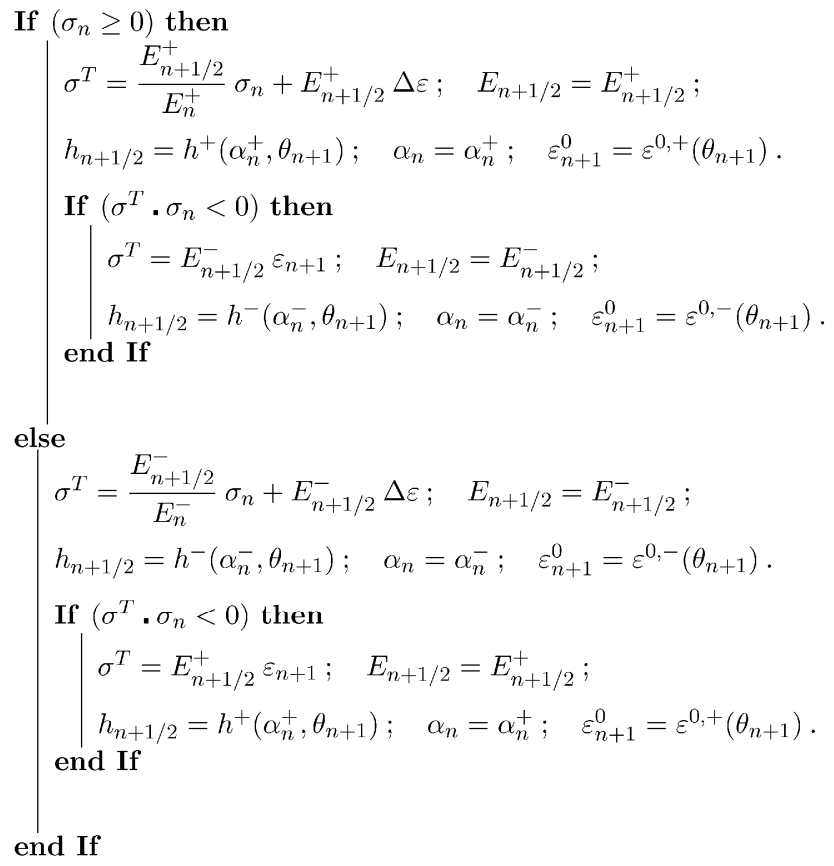

4. Correction phase.

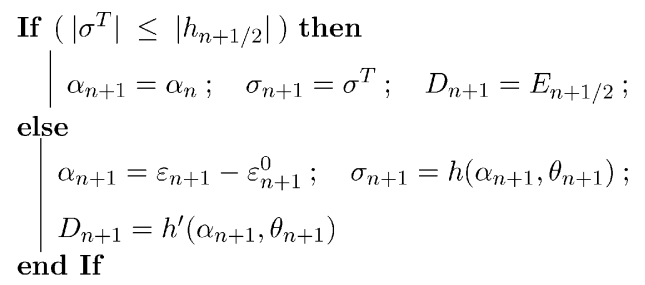

Figure 10: Incremental algorithm for the 3D damage model. 
Table 1: Data table for the tension test.

\begin{tabular}{|c|c|c|c|}
\hline \multicolumn{2}{|c|}{$\begin{array}{c}2500{ }^{\circ} \mathrm{C} \\
\varepsilon^{0}=0 \\
E^{0}=25 \mathrm{GPa}\end{array}$} & \multicolumn{2}{|c|}{$\begin{array}{c}2750^{\circ} \mathrm{C} \\
\varepsilon^{0}=0 \\
E^{0}=12 \mathrm{GPa}\end{array}$} \\
\hline$\varepsilon(\%)$ & $\sigma(\mathrm{MPa})$ & $\varepsilon(\%)$ & $\sigma(\mathrm{MPa})$ \\
\hline 0.0 & 0.0 & 0.0 & 0.0 \\
\hline 0.19 & 44.700 & 0.17 & 19.133 \\
\hline 0.38 & 84.270 & 0.34 & 36.191 \\
\hline 0.57 & 119.024 & 0.51 & 51.294 \\
\hline 0.76 & 149.274 & 0.67 & 64.562 \\
\hline 0.95 & 175.332 & 0.84 & 76.115 \\
\hline 1.14 & 197.511 & 1.01 & 86.074 \\
\hline 1.33 & 216.124 & 1.18 & 94.557 \\
\hline 1.52 & 231.481 & 1.35 & 101.685 \\
\hline 1.71 & 243.898 & 1.52 & 107.579 \\
\hline 1.89 & 253.684 & 1.68 & 112.357 \\
\hline 2.08 & 261.154 & 1.85 & 116.141 \\
\hline 2.27 & 266.619 & 2.02 & 119.050 \\
\hline 2.46 & 270.393 & 2.19 & 121.205 \\
\hline 2.65 & 272.787 & 2.36 & 122.724 \\
\hline 2.84 & 274.114 & 2.53 & 123.729 \\
\hline 3.03 & 274.686 & 2.69 & 124.339 \\
\hline 3.22 & 274.816 & 2.86 & 124.674 \\
\hline 3.41 & 274.817 & 3.03 & 124.854 \\
\hline 3.60 & 275.000 & 3.20 & 125.000 \\
\hline
\end{tabular}

Table 2: The compression test.

\begin{tabular}{|c|c|c|c|}
\hline \multicolumn{2}{|c|}{$\begin{array}{c}2200{ }^{\circ} \mathrm{C} \\
\varepsilon^{0}=0 \\
=69 \mathrm{GPa}\end{array}$} & \multicolumn{2}{|c|}{$\begin{array}{c}2750{ }^{\circ} \mathrm{C} \\
\varepsilon^{0}=0 \\
E^{0}=59 \mathrm{GPa}\end{array}$} \\
\hline$\varepsilon(\%)$ & $\sigma(\mathrm{MPa})$ & $\varepsilon(\%)$ & $\sigma(\mathrm{MPa})$ \\
\hline 0.0 & 0.0 & 0.0 & 0.0 \\
\hline 0.02 & 14.270 & 0.04 & 22.253 \\
\hline 0.04 & 28.034 & 0.08 & 42.511 \\
\hline 0.06 & 41.300 & 0.12 & 60.887 \\
\hline 0.08 & 54.077 & 0.16 & 77.498 \\
\hline 0.11 & 66.373 & 0.20 & 92.459 \\
\hline 0.13 & 78.199 & 0.24 & 105.884 \\
\hline 0.15 & 89.561 & 0.28 & 117.888 \\
\hline 0.17 & 100.469 & 0.32 & 128.586 \\
\hline 0.19 & 110.933 & 0.36 & 138.094 \\
\hline 0.21 & 120.959 & 0.39 & 146.526 \\
\hline 0.23 & 130.558 & 0.43 & 153.998 \\
\hline 0.25 & 139.738 & 0.47 & 160.625 \\
\hline 0.27 & 148.508 & 0.51 & 166.521 \\
\hline 0.29 & 156.876 & 0.55 & 171.802 \\
\hline 0.32 & 164.851 & 0.59 & 176.582 \\
\hline 0.34 & 172.443 & 0.63 & 180.977 \\
\hline 0.36 & 179.659 & 0.67 & 185.101 \\
\hline 0.38 & 186.508 & 0.71 & 189.071 \\
\hline 0.40 & 193.000 & 0.75 & 193.000 \\
\hline
\end{tabular}




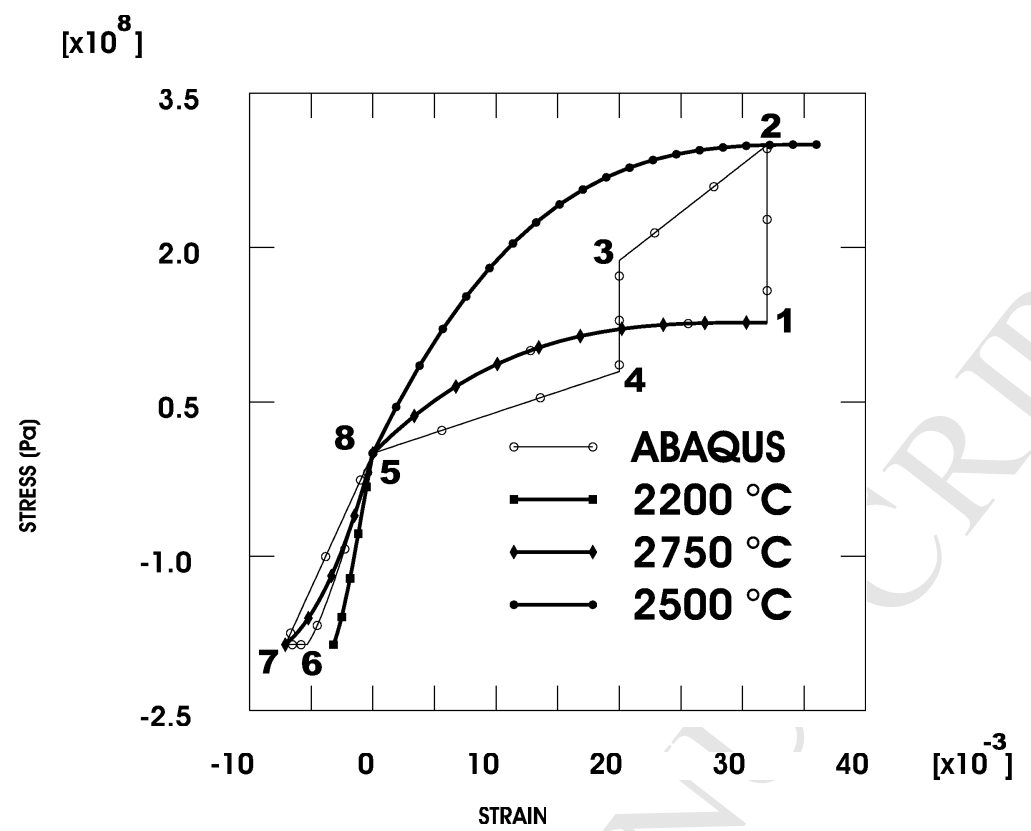

Figure 11: Uniaxial computational test.

\section{AppendixA. Thermodynamic consistency of the model}

Let us write into the framework of the Irreversible Processes Thermodynamics, the simplified (uncoupled) damage model with temperature. The stress response given by model specified in section 6.3 is written

$$
\sigma=D \varepsilon
$$

in the absence of residual strains. The operator $D$ of the elastic stiffness moduli is here diagonal :

$$
\sigma_{i i}=E_{i} \varepsilon_{i i}, \quad \sigma_{i j}=2 G_{i j} \varepsilon_{i j} \quad \text { for } i \neq j .
$$


The operator $D$ depends on $\alpha$ and $\theta$, as also on $s g \varepsilon_{i j}$ where the sign of a quantity $\psi$ is denoted

$$
s g \psi= \begin{cases}+1 & \text { if } \psi \geq 0, \\ -1 & \text { elsewhere }\end{cases}
$$

Constitutive equations (A.1) are written equivalently

$$
\sigma=\rho \frac{\partial w}{\partial \varepsilon},
$$

where $\rho$ is the density independent of $\varepsilon$ and $\alpha$. The notation

$$
\frac{\partial w}{\partial \varepsilon}=\left(\frac{\partial w}{\partial \varepsilon_{i j}}\right)
$$

stands for the gradient of $w$ with respect to $\varepsilon$. The thermodynamic potential $w$ is defined as

$$
w=\frac{1}{\rho}\left\{\frac{1}{2} D \varepsilon: \varepsilon+\bar{w}\right\}
$$

where $\bar{w}$ is a given function of $(\alpha, \theta)$. The specific free energy (Helmotz) $w$ is a differentiable and convex function with respect to $\varepsilon$.

The force variable $a$ associated to $\alpha$ is defined from the gradient of $w$ with respect to $\alpha$ as follows, see Germain et al. (1983):

$$
a=-\rho \frac{\partial w}{\partial \alpha}
$$

The process is thermodynamically admissible if

$$
d \geq 0
$$

by denoting $d=a \cdot \dot{\alpha}$ the dissipation. In the present case, one has

$$
d=-\left\{\frac{1}{2} \frac{\partial D}{\partial \alpha} \varepsilon: \varepsilon+\frac{\partial \bar{w}}{\partial \alpha}\right\} \cdot \dot{\alpha}
$$


that is to say

$$
\begin{aligned}
d= & -\frac{1}{2} \sum_{i}\left\{\frac{\partial E_{i}}{\partial \alpha_{i i}^{+}} \dot{\alpha}_{i i}^{+}+\frac{\partial E_{i}}{\partial \alpha_{i i}^{-}} \dot{\alpha}_{i i}^{-}\right\} \varepsilon_{i i}^{2} \\
& -\sum_{i<j}\left\{\frac{\partial G_{i j}}{\partial \dot{\alpha}_{i j}^{+}} \dot{\alpha}_{i j}^{+}+\frac{\partial G_{i j}}{\partial \alpha_{i j}^{-}} \dot{\alpha}_{i j}^{-}\right\} \varepsilon_{i j}^{2}-\frac{\partial \bar{w}}{\partial \alpha} \cdot \dot{\alpha}
\end{aligned}
$$

The choice of $\bar{w}$ in the expression of the free energy (A.3) determines the force variable $a$ from (A.4). Let us suppose that $\bar{w}$ is a nonincreasing differentiable function with respect to $\alpha_{i j}^{ \pm}$:

$$
\frac{\partial \bar{w}}{\partial \alpha_{i j}^{ \pm}} \leq 0
$$

Under the assumptions (29) and (A.7), the inequality of dissipation (A.5) becomes

$$
\dot{\alpha}_{i j}^{ \pm} \geq 0
$$

by taking into account the expression (A.6) of the dissipation.

\section{References}

Allix, O., Cluzel, C., Ladevèze, P., 1993. Modélisation des composites céramique-céramique à différentes échelles. Revue des composites et des matériaux avancés 3, 277-297.

Chaboche, J.L., 1997. Lois d'évolution de l'endommagement, études de cas. IPSI cours 10, vol. 2.

Desmorat, R., Gatuingt, F., Ragueneau, F., 2010. Nonstandard thermodynamics framework for robust computations with induced anisotropic damage. Int. J. Damage Mechanics 19 (1), 53-73. 
Gasser, A., 1994. Sur la modélisation et l'identification du comportement des composites céramique-céramique à température ambiante. Ph.D. thesis, ENS, Cachan.

Germain, P., Nguyen, Q. S., Suquet, P., 1983. Continuum thermodynamics. J. Appl. Mech. 50, 1010-1020.

Glowinski, R., Lions, J.L., Trémolières, R., 1976. Analyse numérique des inéquations variationnelles. Tome 1 : Théorie générale premières applications. Dunod, Paris.

Laborde, P., to appear. Non-monotone differential inclusions and damage problems.

Laborde, P., Michrafy, A., 1991. On general constitutive equations involving damage. European Journal of Mechanics - A/Solids 10 (2), 213-237.

Ladevèze, P., Gasser, A., Allix, O., 1994. Damage mechanics modeling for ceramic composites. Journal of Engineering Materials and Technology $116(3), 331-336$.

Lemaitre, J., Chaboche, J.L., 1985. Mécanique des matériaux solides. Dunod, Paris.

Maire, J.F., Chaboche, J.L., 1997. A new formulation of continuum damage mechanics (CDM) for composite materials. Aerospace Science and Technology 4, 247-257.

Maire, J.F., Lesné, O., Petipas, C., oct 1996. Une nouvelle approche 
de modélisation du comportement visqueux des composites organiques. Journées nationales sur les composites $N^{\circ} 10$, Paris, France.

Maire, J.F., Lesne, P.M., 1997. A damage model for ceramic matrix composites. Aerospace Science and Technology 4, 259-266.

Moncel, L., 1999. Etude des mécanismes d'endommagement d'un assemblage cuivre/composite carbone-carbone sous chargement thermomécanique. Ph.D. thesis, University of Bordeaux.

Pailhes, J., Camus, G., Lamon, J., 2002. A constitutive model for mechanical behavior of a 3D C/C composite. Mechanics of Materials 34, 161-177.

Poss, M., 1981. Comportement mécanique des composites 3D. Ph.D. thesis, Paris VI University.

Ragueneau, F., Desmorat, R., Gatuingt, F., 2008. Anisotropic damage modelling of biaxial behaviour and rupture of concrete structures. Computers and Concrete 5 (4), 417-434.

Siron, O., 1996. Approche micro-macro du comportement mécanique et de la rupture d'un composite carbone-carbone à architecture fibreuse multidirectionnelle. Ph.D. thesis, University of Bordeaux. 